Document downloaded from:

http://hdl.handle.net/10251/84594

This paper must be cited as:

Gregori Verdú, S.; Tur Valiente, M.; Nadal Soriano, E.; Aguado, J.; Fuenmayor Fernández, FJ.; Chinesta, F. (2017). Fast simulation of the pantograph-catenary dynamic interaction. Finite Elements in Analysis and Design. 129:1-13. doi:10.1016/j.finel.2017.01.007.

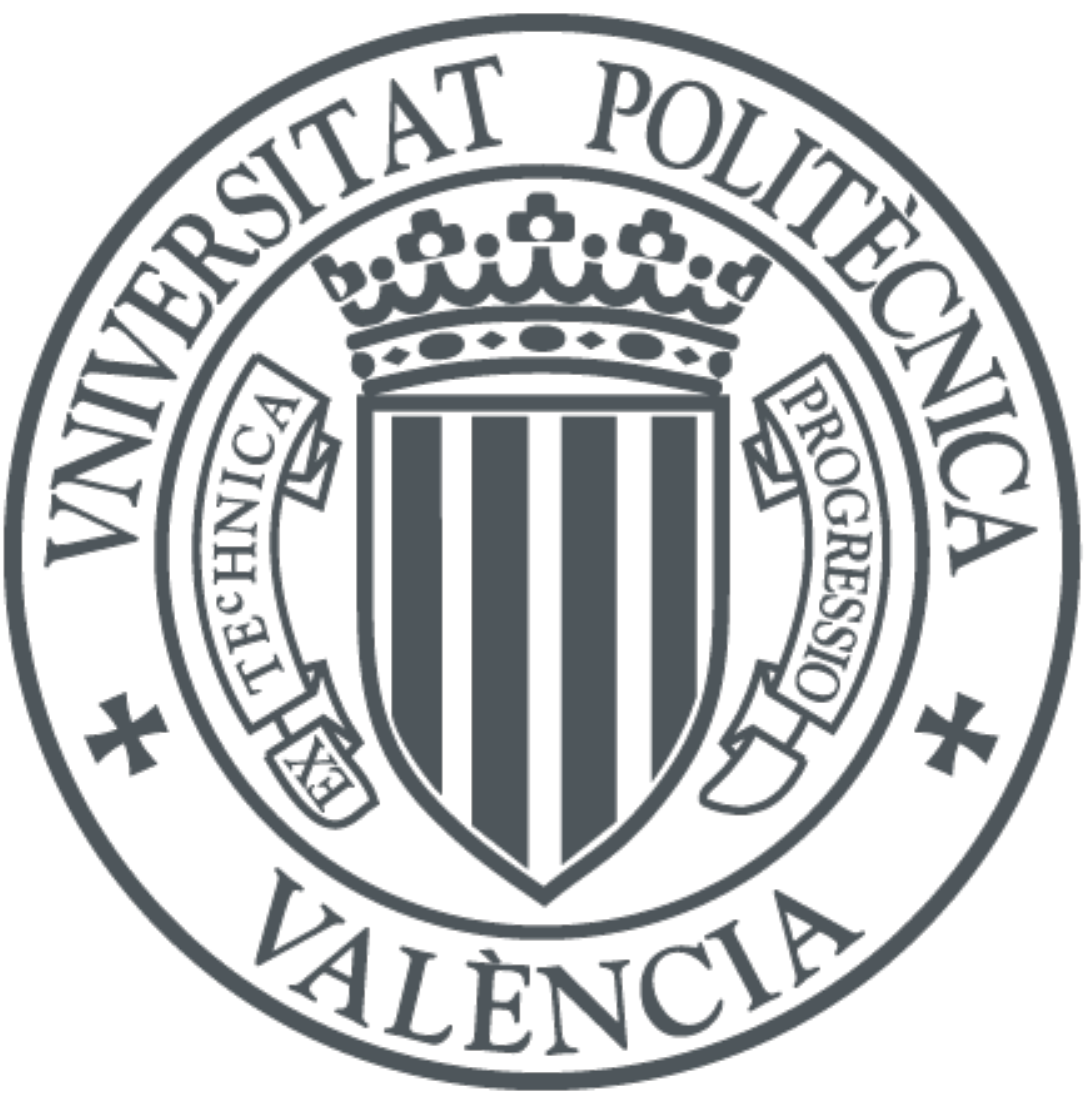

The final publication is available at

http://dx.doi.org/10.1016/j.finel.2017.01.007

Copyright Elsevier

Additional Information 


\title{
Fast simulation of the pantograph-catenary dynamic interaction
}

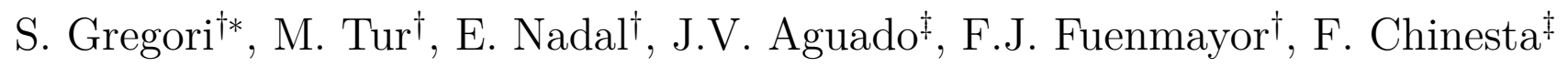 \\ †Centro de Investigación en Ingeniería Mecánica, Universitat Politècnica de València, \\ Camino de Vera s/n, 46022 Valencia, Spain \\ ‡UMR CNRS-Centrale Nantes, 1 rue de la Noe, BP 92101, F-44321 Nantes Cedex 3, France
}

December, 2016

\begin{abstract}
Simulation of the pantograph-catenary dynamic interaction has now become a useful tool for designing and optimizing the system. In order to perform accurate simulations, including system non-linearities, the Finite Element Method is commonly employed combined with a time integration scheme, even though the computational time required may be longer than with the use of other simpler approaches. In this paper we propose a two-stage methodology (Offline/Online) which notably reduces the computational cost without any loss in accuracy and makes it possible to successfully carry out very efficient optimizations or even Hardware in the Loop simulations with real-time requirements.
\end{abstract}

\section{Introduction}

The catenary provides the energy supply to railway locomotives through contact with the pantograph. This overhead equipment is composed of structural elements such as masts,

\footnotetext{
*Corresponding author
} 
brackets and registration arms that hold the contact wire in the desired position. However, other cables form part of the catenary. These include the messenger wire, which is hooked to the brackets, and the droppers, which suspend the contact wire from the messenger wire.

Current collection quality is vital for good performance and is usually measured by the pantograph-catenary interaction force. High contact forces cause high levels of wear on the sliding surfaces [1], while contact losses produce arcing and cut out the energy supply to the engines [2], limiting the operational railway speed. This means numerical simulations can be very useful in the design of improved pantographs and overhead equipment.

In recent decades a lot of effort has been put into the simulation of the pantographcatenary dynamic interaction (see [3] and the references therein), and has triggered European projects (EUROPAC) and regulation in the field [4] (UNE 50318). Starting with very simple models [5, 6], the Finite Element Method (FEM) now seems to be the most suitable approach for modelling the overhead line [7]. Pantograph mathematical modelling ranges from linear lumped parameter models to flexible multibody models [8].

Computational cost is always an issue if simulations have to be performed several times as occurs in optimization procedures. In the catenary-pantograph field some authors have proposed simplified catenary models [9], modal decomposition approaches [10], models based on moving meshes [11] or even a priori model order reduction techniques [12] in order to reduce the computational effort. However, the common feature of all these approaches is that they are less accurate than the full FE models.

At the present time, hybrid or Hardware In the Loop (HIL) simulations are another field in which the computational cost is crucial. The main requirement of such approaches is a numerical model that can be solved in real-time. A wide range of applications can be found in many fields, such as in earthquake engineering [13], electronic engineering [14] and railway mechanics [15] to cite just a few. When testing pantographs, it is advisable to have an accurate catenary model. Although HIL simulation techniques have been implemented for pantograph-catenary dynamic interaction (see the pioneering works $[10,16]$ ), even though they all achieve real-time performance, a somewhat simplified catenary model with a modal approach is generally used.

This paper aims to provide a realistic model of the pantograph-catenary dynamic in- 
teraction that can be solved efficiently without losing any of the accuracy achieved by classical FE techniques. The computational cost saves can be exploited to perform efficient optimizations and furthermore, the method is suitable to be used in HIL simulations if real-time response is achieved. Extending on the idea first introduced by Collina and Bruni in [17] and used by Ambrósio et al. in [30], in which the non-linearities of the model are moved to the right hand side of the equation of motion, the main novelty of the method proposed here relies on pre-calculating as much information as possible in an Offline stage. In this phase of the calculations, the catenary is treated as a linear system, and then in the subsequent Online stage the non-linearities introduced by dropper slackening and loss of pantograph-catenary contact are dealt with. The pre-calculated information makes it possible to obtain the total system response by solving a system with a small set of unknowns, which notably reduces the computational cost required for the time integration.

The paper is organized as follows. After this brief introduction, the numerical models chosen for the different subsystems are described in the next section. The static equilibrium formulation is a requirement for the dynamic simulation and is detailed in Section 3. In Section 4 we explain in detail the dynamic interaction problem. The features of the new Offline/Online approach are deduced from what we call the classical time integration procedure and its modified versions. Some numerical examples are given in Section 5 to validate the assumed hypotheses and also to show the saving in simulation time of the proposed method. Finally, we provide some concluding remarks in Section 6.

\section{Catenary and pantograph models}

In this section we describe all the models used to perform the numerical simulations of the pantograph-catenary dynamic interaction. There are three different subsystems which need to be modelled: the catenary, the pantograph and the interaction between these two subsystems. 


\subsection{Catenary model}

Among the different options found in the literature, the FEM is the method most frequently used to model realistic catenary behaviour. In the present work the catenary cables are modelled by a beam element based on the Absolute Nodal Coordinate Formulation (ANCF) first proposed by Shabana [18] and adapted for thin beams and cables in [19]. This formulation has also been used by other authors for railway catenary models $[20,21]$. The interested reader is referred to [22, 23] for detailed comparisons between the ANCF element and those based on the classical formulation.

For very slender beams like catenary cables, the ANCF element has only 6 degrees of freedom per node in 3D, taking into account axial and bending deformations. Hereinafter, this type of element is referred as 'cable element' and is used to model both the messenger and the contact wires. Droppers and registration arms are modelled as a single large displacement non-linear element, known as the 'bar element' throughout the paper. All

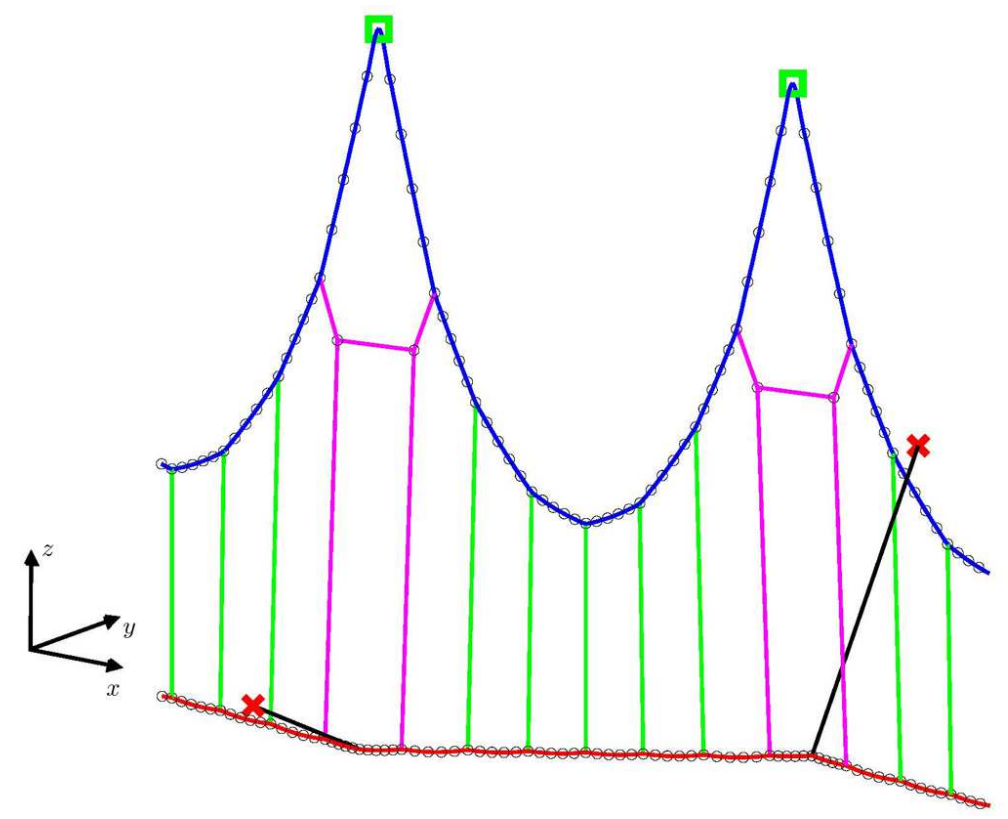

Figure 1: FE catenary model with boundary conditions.

the supports are replaced by suitable boundary conditions. The displacements at the ends of the registration arms joined to the brackets (nodes marked with a cross in Fig. 1) are constrained. 

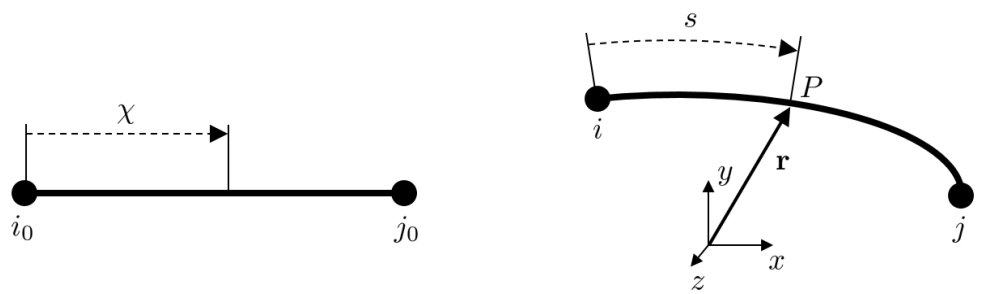

Figure 2: Reference and deformed configurations of the ANCF element.

In Fig. 2 the reference and deformed configurations for a cable element is schematically represented. The vector of degrees of freedom for an element with nodes $i$ and $j$ is:

$$
\mathbf{q}_{c}=\left[\begin{array}{llllllllllll}
x_{i} & y_{i} & z_{i} & \frac{\partial x_{i}}{\partial \chi} & \frac{\partial y_{i}}{\partial \chi} & \frac{\partial z_{i}}{\partial \chi} & x_{j} & y_{j} & z_{j} & \frac{\partial x_{j}}{\partial \chi} & \frac{\partial y_{j}}{\partial \chi} & \frac{\partial z_{j}}{\partial \chi}
\end{array}\right]^{T}
$$

where $\chi \in\left[0, l_{\text {ref }}\right]$ is the local coordinate, $l_{\text {ref }}$ is the initial length of the element, $x_{i}, y_{i}, z_{i}$ are the coordinates of node $i$ and $\frac{\partial x_{i}}{\partial \chi}, \frac{\partial y_{i}}{\partial \chi}, \frac{\partial z_{i}}{\partial \chi}$ are the slopes. In a deformed configuration, the absolute position at a given point with local coordinate $\chi$ is defined by means of a cubic Hermitian interpolation that can be written as:

$$
\mathbf{r}(\chi)=\mathbf{N}_{c}(\chi) \mathbf{q}_{c}
$$

where

$$
\begin{gathered}
\mathbf{N}_{c}(\chi)=\left[N_{c 1} \mathbf{I}_{3}\left|N_{c 2} \mathbf{I}_{3}\right| N_{c 3} \mathbf{I}_{3} \mid N_{c 4} \mathbf{I}_{3}\right] \\
N_{c 1}(\xi)=1-3 \xi^{2}+2 \xi^{3} \quad N_{c 2}(\xi)=l_{r e f}\left(\xi-2 \xi^{2}+\xi^{3}\right) \\
N_{c 3}(\xi)=3 \xi^{2}-2 \xi^{3} \quad N_{c 4}(\xi)=l_{\text {ref }}\left(-\xi^{2}+\xi^{3}\right)
\end{gathered}
$$

The coordinate $\xi=\chi / l_{\text {ref }} \in[0,1]$ denotes the normalized local coordinate and $\mathbf{I}_{3}$ is the $3 \times 3$ identity matrix. The $C^{1}$ continuity of the solution between elements is guaranteed with this interpolation. The degrees of freedom of a bar element are the absolute positions of the two nodes of the element only, namely:

$$
\mathbf{q}_{b}=\left[\begin{array}{llllll}
x_{i} & y_{i} & z_{i} & x_{j} & y_{j} & z_{j}
\end{array}\right]^{T}
$$

In these elements, as no bending deformations are taken into account, a linear interpola- 
tion is enough to ensure continuity of the solution,

$$
\begin{gathered}
\mathbf{N}_{b}(\chi)=\left[N_{b 1} \mathbf{I}_{3} \mid N_{b 2} \mathbf{I}_{3}\right] \\
N_{b 1}(\xi)=-\frac{\xi-1}{2} \quad N_{b 2}(\xi)=\frac{\xi+1}{2}
\end{gathered}
$$

For simplicity in the notation, subscripts $c$ and $b$ will not appear henceforth unless necessary.

\subsection{Pantograph model}

A number of accurate pantograph models can be found in the bibliography. Although these models are based on FEM or multibody approaches [8, 24] and are able to account for deformable bodies, the most widely used pantograph model in the literature is a lumped mass model with 2 or 3 vertical degrees of freedom. We have assumed a model with 3 masses, which is depicted in Fig. 3.

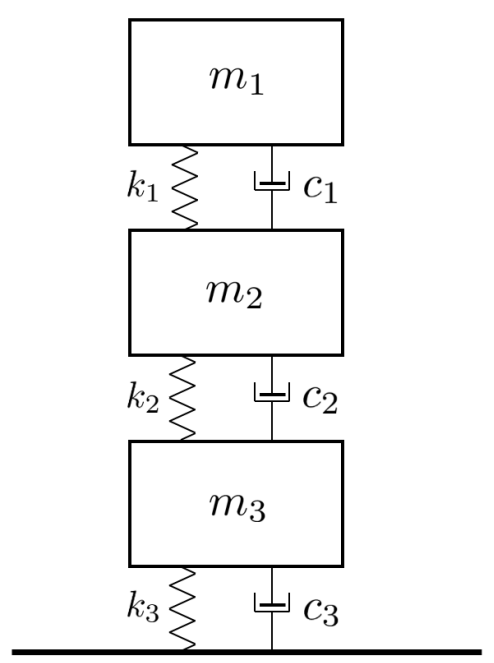

Figure 3: Lumped mass pantograph model. 


\subsection{Interaction model}

In order to model the pantograph-catenary interaction, approaches like the imposition of unilateral constraints by Lagrange multipliers [25] or an Hertzian type contact force with internal damping [26] are found in the literature. However, in this work the pantographcatenary interaction is simulated by a simple and widely used penalty method. This method introduces a high stiffness elastic element which connects the pantograph head with the contact wire in order to accomplish the impenetrability constraint. A scheme of this type of interaction is represented in Fig. 4. According to the reference model in [4], the value of the penalty constant is set at $k_{h}=50000 \mathrm{~N} / \mathrm{m}$. The contact or interaction force vector is assumed to be oriented vertically and its value can be computed as:

$$
f_{i}= \begin{cases}k_{h}\left(z_{1}-z_{c w}\right) & \text { if } z_{1} \geq z_{c w} \\ 0 & \text { if } z_{1}<z_{c w}\end{cases}
$$

where $z_{1}$ and $z_{c w}$ are the vertical absolute coordinates of the mass 1 of the pantograph and the contact point on the contact wire respectively.

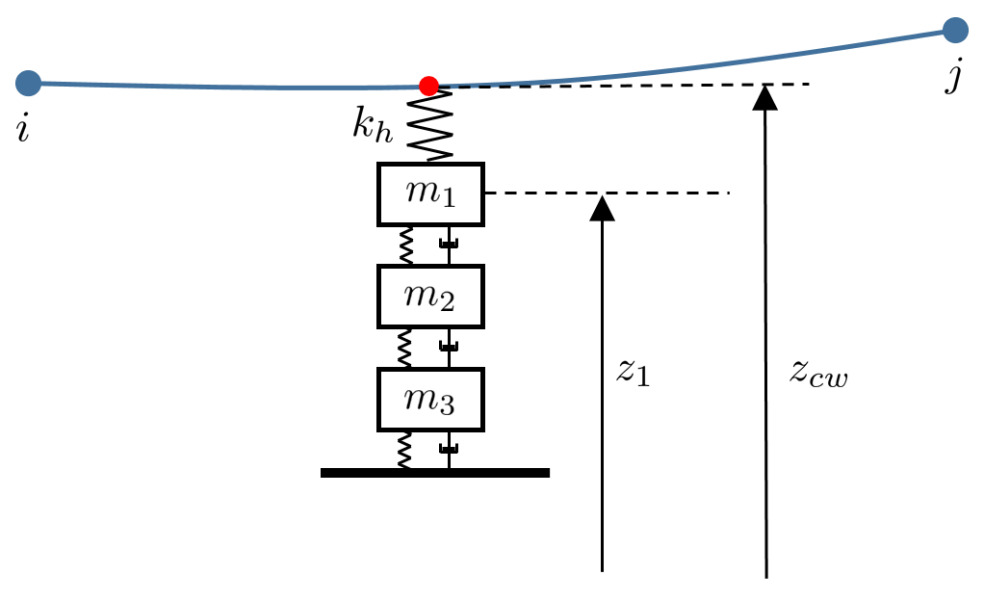

Figure 4: Pantograph-catenary interaction scheme. 


\section{Static equilibrium configuration}

As in any cable structure, the first step in the simulation consists of solving the so-called shape finding problem. During catenary stringing certain constraints must be fulfilled by the cabling. In the problem at hand, the main constraints are the initial tensions of the messenger and contact wires, contact wire height and the separation between droppers. In this initial configuration problem, the non-deformed element lengths and the nodal degrees of freedom are therefore set as the unknowns. A detailed explanation of this problem is thoroughly discussed in [27].

Here we assume that the non-deformed element lengths are known and that $\Omega$ represents the spatial domain of the catenary. This domain is discretised into $N_{e}$ non-overlapping elements with a domain $\Omega^{e}$ that fulfil $\Omega=\bigcup_{e=1}^{N_{e}} \Omega^{e}$. The virtual work principle states that the virtual work produced by the internal forces must be equal to the virtual work produced by the external ones,

$$
\sum_{e=1}^{N_{e}} \delta W_{i n t}^{e}-\delta W_{e x t}^{e}=0
$$

For a certain element $e$ belonging to a cable with cross-sectional area $A$, Young's modulus $E$ and second moment of area $I$, the internal virtual work is produced by a combination of axial and bending strains:

$$
\delta W_{i n t}^{e}=\int_{\Omega^{e}}\left(E A \delta \varepsilon_{a} \varepsilon_{a}+E I \delta \kappa \kappa\right) d \chi
$$

where $\varepsilon_{a}$ is the axial strain and $\kappa$ is the curvature. During catenary stringing, the cables undergo large displacements, so that a non-linear measure of the deformation is required. Using the Green strain tensor, the axial deformation is defined as:

$$
\varepsilon_{a}=\frac{1}{2}\left(\frac{d \mathbf{r}}{d \chi} \cdot \frac{d \mathbf{r}}{d \chi}-1\right)
$$

Since the cables undergo small deformations, we can assume $d s \approx d \chi$ and therefore 
curvature can be approximated by [28]:

$$
\kappa \approx\left|\frac{d^{2} \mathbf{r}}{d \chi^{2}}\right|
$$

The only external force applied to the system is the force of gravity. The virtual work produced by this force is:

$$
\delta W_{e x t}^{e}=\int_{\Omega^{e}} \delta \mathbf{r} \cdot \mathbf{g} d \chi
$$

where the vector $\mathbf{g}=\left[\begin{array}{lll}0 & 0 & -g A \rho\end{array}\right]^{T}$, while $g$ is the gravitational constant and $\rho$ is the density of the cable. By combining all the previous expressions, the static equilibrium problem consists of finding the field $\mathbf{r}(\chi)$ for any admissible $\delta \mathbf{r}$, such that:

$$
\sum_{e=1}^{N_{e}} \int_{\Omega^{e}}\left[E I \frac{d^{2} \delta \mathbf{r}}{d \chi^{2}} \cdot \frac{d^{2} \mathbf{r}}{d \chi^{2}}+\frac{E A}{2} \frac{d \delta \mathbf{r}}{d \chi} \cdot \frac{d \mathbf{r}}{d \chi}\left(\frac{d \mathbf{r}}{d \chi} \cdot \frac{d \mathbf{r}}{d \chi}-1\right)-\delta \mathbf{r} \cdot \mathbf{g}\right] d \chi=0, \quad \forall \delta \mathbf{r}
$$

where the first term related with bending strain vanishes for the bar elements since $I=0$ for them.

After the assembly process, taking into account all the Dirichlet boundary conditions, the non-linear algebraic system of equations

$$
\mathbf{f}_{\text {int }}(\mathbf{q})-\mathbf{f}_{e x t}=\mathbf{0}
$$

is obtained, which can be solved using for example the Newton-Raphson method.

\section{Dynamic interaction problem}

\section{Catenary}

The dynamic behaviour of the catenary system is characterized by the small cable displacements. A common assumption is to linearise the dynamic problem with respect to the static equilibrium position of the catenary cables.

Once the static equilibrium position of the catenary cabling $\mathbf{r}_{0}$ has been calculated by

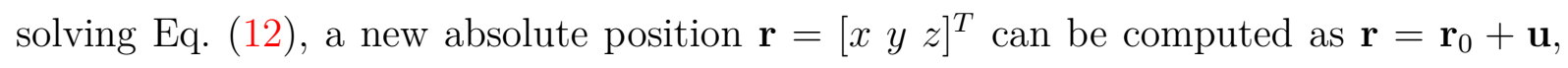


where $\mathbf{u}=\left[\begin{array}{lll}u & v & w\end{array}\right]^{T}$ are the displacements with respect to the static equilibrium position. Note that subscript 0 refers to the configuration for which the system has been linearised.

If Eq. (12) is linearised and we add the inertial term, the linear dynamic problem for the catenary consists of finding $\mathbf{u}$ for any compatible $\delta \mathbf{u}$ such that:

$$
\begin{gathered}
\sum_{e=1}^{N_{e}} \int_{\Omega^{e}}\left[\rho A \delta \mathbf{u} \cdot \ddot{\mathbf{u}}+E I \frac{d^{2} \delta \mathbf{u}}{d \chi^{2}} \cdot \frac{d^{2} \mathbf{u}}{d \chi^{2}}+\frac{E A}{2} \frac{d \delta \mathbf{u}}{d \chi} \cdot\left[2 \frac{d \mathbf{r}_{0}}{d \chi}\left(\frac{d \mathbf{u}}{d \chi} \cdot \frac{d \mathbf{r}_{0}}{d \chi}\right)+\right.\right. \\
\left.\left.\frac{d \mathbf{u}}{d \chi}\left(\frac{d \mathbf{r}_{0}}{d \chi} \cdot \frac{d \mathbf{r}_{0}}{d \chi}-1\right)\right]\right] d \chi=0, \quad \forall \delta \mathbf{u}
\end{gathered}
$$

Introducing the interpolations defined in Section 2.1 for each type of element, the mass and stiffness matrices of the catenary are:

$$
\begin{aligned}
& \mathbf{M}_{\text {cat }}= \mathrm{A}_{e} \int_{\Omega^{e}} \rho A \mathbf{N}^{T} \mathbf{N} d \chi \\
& \mathbf{K}_{c a t}=\mathbf{A}_{e} \int_{\Omega^{e}}\left[E I \mathbf{N}^{\prime \prime} \mathbf{N}^{\prime \prime}+E A\left(\left(\mathbf{N}^{\prime T} \mathbf{N}^{\prime} \mathbf{q}_{0}\right)\left(\mathbf{q}_{0}^{T} \mathbf{N}^{\prime T} \mathbf{N}^{\prime}\right)+\right.\right. \\
&\left.\left.\frac{1}{2}\left(\mathbf{N}^{\prime T} \mathbf{N}^{\prime}\right)\left(\mathbf{q}_{0}^{T} \mathbf{N}^{\prime T} \mathbf{N}^{\prime} \mathbf{q}_{0}\right)-\frac{1}{2} \mathbf{N}^{\prime T} \mathbf{N}^{\prime}\right)\right] d \chi
\end{aligned}
$$

where $\mathbf{A}$ is the assembly operator, $\mathbf{N}$ is the shape functions matrix for both cable and bar elements whose derivatives are $\mathbf{N}^{\prime}=\frac{d \mathbf{N}}{d \chi}$ and $\mathbf{N}^{\prime \prime}=\frac{d^{2} \mathbf{N}}{d \chi^{2}}$.

In addition to this linearisation, if we analyse Eq. (14) in more detail, another simplification can be introduced for the dropper elements. The vector $\frac{d \mathbf{r}_{0}}{d \chi}=\mathbf{n}_{d}$ has the direction of the dropper in the configuration in which the problem has been linearised (i.e. the static equilibrium position) and its modulus is $\frac{l_{0}}{l_{r e f}}$. Therefore, the virtual work produced by axial strains can be decomposed into two terms. For a single dropper element, the first of them reads:

$$
\int_{\Omega^{e}} E A\left(\frac{d \delta \mathbf{u}}{d \chi} \cdot \mathbf{n}_{d}\right)\left(\frac{d \mathbf{u}}{d \chi} \cdot \mathbf{n}_{d}\right) d \chi=\int_{\Omega^{e}} E A\left(\frac{l_{0}}{l_{r e f}}\right)^{2} \frac{d \delta \mathbf{u}}{d \chi}\left(\hat{\mathbf{n}}_{d} \otimes \hat{\mathbf{n}}_{d}\right) \frac{d \mathbf{u}}{d \chi} d \chi
$$

where the unitary vector $\hat{\mathbf{n}}_{d}=\frac{\mathbf{n}_{d}}{\left\|\mathbf{n}_{d}\right\|}$. The second term is:

$$
\int_{\Omega^{e}} \frac{E A}{2} \frac{d \delta \mathbf{u}}{d \chi} \cdot \frac{d \mathbf{u}}{d \chi}\left(\left(\frac{l_{0}}{l_{\text {ref }}}\right)^{2}-1\right) d \chi=\int_{\Omega^{e}} E A \varepsilon_{a, 0} \frac{d \delta \mathbf{u}}{d \chi} \cdot \frac{d \mathbf{u}}{d \chi} d \chi=\int_{\Omega^{e}} T_{0} \frac{d \delta \mathbf{u}}{d \chi} \cdot \frac{d \mathbf{u}}{d \chi} d \chi
$$


where $\varepsilon_{a, 0}$ and $T_{0}$ are the axial strain and the tension in the static equilibrium position respectively.

If $T_{0} \ll E A\left(\frac{l_{0}}{l_{r e f}}\right)^{2}$, the components of (17) aligned with the dropper can be neglected. Furthermore, assuming that the movements in the perpendicular directions to the dropper are small (what is not applicable for the messenger and contact wires), the fully contribution of the term (17) is negligible and therefore, the simplified stiffness matrix for a dropper element is:

$$
\mathbf{k}_{d}=\int_{\Omega^{e}} E A\left(\frac{l_{0}}{l_{\text {ref }}}\right)^{2} \mathbf{N}_{b}^{\prime T} \hat{\mathbf{n}}_{d}^{T} \hat{\mathbf{n}}_{d} \mathbf{N}_{b}^{\prime} d \chi
$$

Once the inertial and elastic properties of the catenary have been defined, proportional Rayleigh damping is also introduced. This leads to a catenary damping matrix $\mathbf{C}_{c a t}=$ $\alpha_{r} \mathbf{M}_{c a t}+\beta_{r} \mathbf{K}_{c a t}$, where $\alpha_{r}$ and $\beta_{r}$ are the damping parameters. Finally, the matrix form of the linear dynamic equations of the catenary system is:

$$
\mathbf{M}_{c a t} \ddot{\mathbf{u}}_{c a t}+\mathbf{C}_{c a t} \dot{\mathbf{u}}_{c a t}+\mathbf{K}_{c a t} \mathbf{u}_{c a t}=\mathbf{0}
$$

\section{Pantograph}

The lumped mass model chosen for the pantograph is linear and it only introduces vertical degrees of freedom. Applying the Lagrange equation of motion to the system, we obtain:

$$
\begin{aligned}
m_{1} \ddot{w}_{1}+c_{1}\left(\dot{w}_{1}-\dot{w}_{2}\right)+k_{1}\left(w_{1}-w_{2}\right) & =0 \\
m_{2} \ddot{w}_{2}+c_{1}\left(\dot{w}_{2}-\dot{w}_{1}\right)+c_{2}\left(\dot{w}_{2}-\dot{w}_{3}\right)+k_{1}\left(w_{2}-w_{1}\right)+k_{2}\left(w_{2}-w_{3}\right) & =0 \\
m_{3} \ddot{w}_{3}+c_{2}\left(\dot{w}_{3}-\dot{w}_{2}\right)+c_{3} \dot{w}_{3}+k_{2}\left(w_{2}-w_{3}\right)+k_{3} w_{3} & =F_{p a n}
\end{aligned}
$$

where $w_{i}, i=1,2,3$ denotes the displacement of the lumped masses with respect to the equilibrium position $z_{\text {ref }}$. This position is defined as the $z_{0}$ (third component of $\mathbf{r}_{0}$ ) coordinate of the initial interaction point in the catenary (see Fig. 4). $F_{\text {pan }}$ is the external uplift force applied to the mass 3 of the pantograph. Besides, all three masses of the pantograph are assumed to be in equilibrium at $z_{\text {ref }}$. In matrix form, the previous equations become:

$$
\mathbf{M}_{\text {pan }} \ddot{\mathbf{w}}_{\text {pan }}+\mathbf{C}_{\text {pan }} \dot{\mathbf{w}}_{\text {pan }}+\mathbf{K}_{\text {pan }} \mathbf{w}_{\text {pan }}=\mathbf{F}_{\text {pan }}
$$




\section{Interaction}

The interaction force $f_{i}$ depends on the position of mass $1 z_{1}$ and the contact wire height $z_{c w}$ which varies as the train moves. Using a penalty method, the virtual work produced by the interaction force is:

$$
\delta z_{i} f_{i}=\left(\delta z_{1}-\delta z_{c w}\right) k_{h}\left(z_{1}-z_{c w}\right)=\left(\delta w_{1}-\delta w_{c w}\right) k_{h}\left(z_{r e f}+w_{1}-z_{0, c w}-w_{c w}\right)
$$

where $k_{h}$ is the penalty constant defined in Section 2.3. The subindex $c w$ shows that the variable is particularized at the interaction point on the contact wire. From now on, if one variable requires more than one subindex they will be separated by a comma.

From Eq. (22), the interaction stiffness matrix, which couples the catenary and pantograph degrees of freedom, is:

$$
\mathbf{k}_{i}=\left(\begin{array}{ll}
\mathbf{k}_{i, c c} & \mathbf{k}_{i, c p} \\
\mathbf{k}_{i, p c} & k_{i, p p}
\end{array}\right)=k_{h}\left(\begin{array}{cccc:c}
N_{c 1}^{2} & N_{c 1} N_{c 2} & N_{c 1} N_{c 3} & N_{c 1} N_{c 4} & -N_{c 1} \\
N_{c 2} N_{c 1} & N_{c 2}^{2} & N_{c 2} N_{c 3} & N_{c 2} N_{c 4} & -N_{c 2} \\
N_{c 3} N_{c 1} & N_{c 3} N_{c 2} & N_{c 3}^{2} & N_{c 3} N_{c 4} & -N_{c 3} \\
N_{c 4} N_{c 1} & N_{c 4} N_{c 2} & N_{c 4} N_{c 3} & N_{c 4}^{2} & -N_{c 4} \\
--- & --- & --- & --- & --- \\
-N_{c 1} & -N_{c 2} & -N_{c 3} & -N_{c 4} & 1
\end{array}\right)
$$

where all the shape functions are evaluated in the local coordinate of the contact wire cable element particularized at the interaction point. Furthermore, a force that only depends on the static equilibrium position from Eq. (22) has also to be considered:

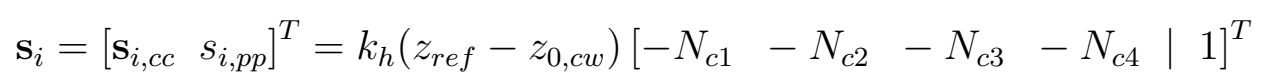

At this point, all the matrices are available to be combined, leading to the semidiscrete dynamic equations of the whole system. If the global vector of displacements is defined as $\mathbf{u}=\left[\begin{array}{ll}\mathbf{u}_{\text {cat }} & \mathbf{w}_{\text {pan }}\end{array}\right]^{T}$, and the different matrices $\mathbf{m}, \mathbf{c}, \mathbf{k}, \mathbf{f}$ and $\mathbf{s}$ presented above are assembled, the dynamic equation of the global system is:

$$
\mathbf{M u ̈}+\mathbf{C} \dot{\mathbf{u}}+\mathbf{K u}=\mathbf{F}
$$


where:

$$
\begin{aligned}
\mathbf{M} & =\left(\begin{array}{cc}
\mathbf{M}_{c a t} & \mathbf{0} \\
\mathbf{0} & \mathbf{M}_{\text {pan }}
\end{array}\right) \\
\mathbf{C} & =\left(\begin{array}{cc}
\mathbf{C}_{c a t} & \mathbf{0} \\
\mathbf{0} & \mathbf{C}_{p a n}
\end{array}\right) \\
\mathbf{K} & =\left(\begin{array}{cc}
\mathbf{K}_{c a t} & \mathbf{0} \\
\mathbf{0} & \mathbf{K}_{p a n}
\end{array}\right)+\left(\begin{array}{ll}
\mathbf{K}_{i, c c} & \mathbf{K}_{i, c p} \\
\mathbf{K}_{i, p c} & \mathbf{K}_{i, p p}
\end{array}\right) \\
\mathbf{F} & =\left(\begin{array}{c}
\mathbf{0} \\
\mathbf{F}_{p a n}
\end{array}\right)-\left(\begin{array}{l}
\mathbf{S}_{i, c c} \\
\mathbf{S}_{i, p p}
\end{array}\right)
\end{aligned}
$$

and the capital letters denote that the variable has been expanded to the global size.

The initial conditions necessary to begin the time integration are now obtained by solving the small displacements linearised problem with the uplift force $\mathbf{F}_{p a n}$ as external force. Hence, $\mathbf{u}^{0}$ is found after solving the linear system of equations $\mathbf{K u}^{0}=\mathbf{F}$. When the pantograph pushes up the catenary some droppers may be compressed. In order to consider the effect of dropper slackening we apply here the same iterative procedure as will be explained in Section 4.1. Finally, since the whole system is at rest at the initial time, $\dot{\mathbf{u}}^{0}$ and $\ddot{\mathbf{u}}^{0}$ are null.

\subsection{Classical time integration procedure}

In order to solve Eq. (25) and therefore obtain the displacements, velocities and accelerations of the whole system, we use the Hilber-Hughes-Taylor (HHT) time integration scheme [29]. This time integrator can be seen as a generalization of the well-known Newmark method. It uses a constant time step $\Delta t$ and some parameters $\alpha, \beta$ and $\gamma$ which control the stability and the numerical damping introduced by the method.

We assume a bilinear behaviour of the droppers, as shown in Fig. 5, where the forceelongation curve for a dropper $d$ of the linearised catenary is depicted. In this figure, $f_{d}$ represents the internal force of the dropper while $s_{d}$ is the value of its traction force in the static equilibrium configuration, i.e. in the configuration in which the equations are linearised. The horizontal axis denotes the elongation of the dropper, $\delta_{0, d}$ being the 
value of elongation at the static equilibrium position. It is also shown that in traction the dropper presents stiffness $k_{d}$, while in compression it is null.

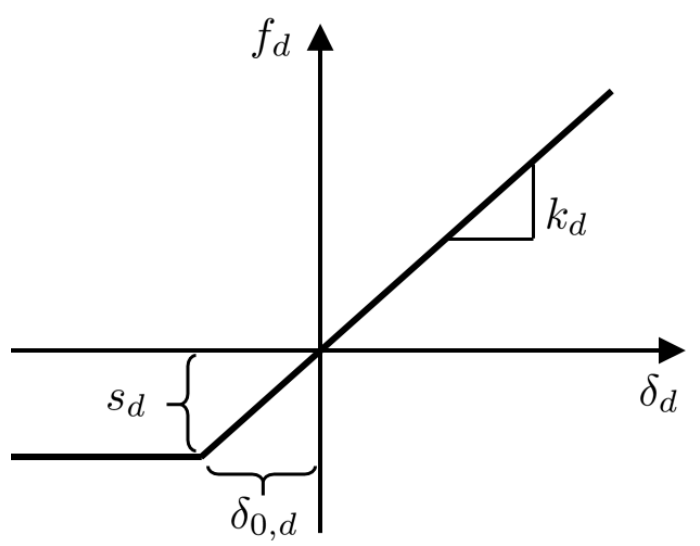

Figure 5: Force-elongation curve for dropper $d$ in the linearised problem.

This bilinear behaviour requires the use of an iterative scheme in order to obtain the solution in each time step. Moreover, an additional non-linearity appears from the fact that the pantograph can be detached from the contact wire with the interaction force vanishing. By applying the HHT algorithm to Eq. (25), as described in Appendix A, the displacements at time step $t, t=1, \ldots, N_{s t p}$, and iteration $j$ are obtained by solving the following linear system of equations:

$$
\mathbf{A}_{j}^{t} \mathbf{u}_{j}^{t}=\mathbf{b}_{j}^{t}
$$

where

$$
\begin{aligned}
\mathbf{A}_{j}^{t} & =\left[(1+\alpha)\left[\mathbf{K}_{j}^{t}+b_{4} \mathbf{C}_{j}^{t}\right]+b_{1} \mathbf{M}\right] \\
\mathbf{b}_{j}^{t} & =-\alpha \mathbf{F}^{t-1}+(1+\alpha) \mathbf{F}_{j}^{t}+\mathbf{F}_{I C}^{t}
\end{aligned}
$$

being

$$
\begin{aligned}
\mathbf{F}_{I C}^{t}= & \alpha\left(\mathbf{K}^{t-1} \mathbf{u}^{t-1}+\mathbf{C}^{t-1} \dot{\mathbf{u}}^{t-1}\right)+\mathbf{M}\left(b_{1} \mathbf{u}^{t-1}-b_{2} \dot{\mathbf{u}}^{t-1}-b_{3} \ddot{\mathbf{u}}^{t-1}\right)+ \\
& (1+\alpha) \mathbf{C}_{j}^{t}\left(b_{4} \mathbf{u}^{t-1}-b_{5} \dot{\mathbf{u}}^{t-1}-b_{6} \ddot{\mathbf{u}}^{t-1}\right)
\end{aligned}
$$

All the $b_{i}, i=1, \ldots, 6$ depend on the time step and the method's parameters $\alpha, \beta$ and $\gamma$. Once $\mathbf{u}_{j}^{t}$ has been obtained we have to evaluate the state of any dropper. The internal 
force at node $n$ of dropper $d$ is $\mathbf{f}_{d, j}^{t}=\left[\mathbf{k}_{d} \mathbf{u}_{d, j}^{t}\right]_{n}$, where the operator $[\cdot]_{n}$ selects only the components of the force applied at the node $n$ of the dropper. This vector is aligned with $\mathbf{s}_{d}$ due to the use of the simplified stiffness matrix considered in (18). Projecting it in the direction of dropper $d$ in the static equilibrium configuration $\mathbf{n}_{d}$ (which is equivalent to calculating its modulus) we obtain $f_{d, j}^{t}$. The slackening criterion is then:

$$
\begin{aligned}
& \text { if } f_{d, j}^{t}+s_{d} \leq 0 \text { then dropper } d \text { is slackened } \\
& \text { if } f_{d, j}^{t}+s_{d}>0 \text { then dropper } d \text { is tensioned }
\end{aligned}
$$

from which the slackening state $\mathscr{D}$ is defined.

The elemental stiffness $\mathbf{k}_{d}$ and damping $\mathbf{c}_{d}$ matrices of the slackened droppers have to be removed from the global ones, and also the internal force of the element in the static equilibrium position must be included in the next iteration $j+1$ in order to account for this state change. Then the given changes are:

$$
\begin{aligned}
& \mathbf{K}_{j+1}^{t}=\mathbf{K}^{t}-\sum_{d}^{N_{s d}} \mathbf{K}_{d} \\
& \mathbf{C}_{j+1}^{t}=\mathbf{C}^{t}-\sum_{d}^{N_{s d}} \mathbf{C}_{d} \\
& \mathbf{F}_{j+1}^{t}=\mathbf{F}^{t}+\sum_{d}^{N_{s d}} \mathbf{S}_{d}
\end{aligned}
$$

where $N_{s d}$ is the total number of slackened droppers. These modifications imply changes in both, the global time-step matrix $\mathbf{A}_{j}^{t}$ and the right hand side of Eq. (27) $\mathbf{b}_{j}^{t}$ at every iteration.

Before moving to the next iteration, the value of the interaction force $f_{i}$ must be obtained from Eq. (22) and then we enforce the contact loss criterion which defines the state $\mathscr{C}$. This consists of setting the contact force to 0 if its value is negative, i.e. if there is contact loss, or leaving it unchanged otherwise.

Now we are ready to start the next iteration $j+1$, compute $\mathbf{u}_{j+1}^{t}$, check the slackening criterion on each dropper, recalculate the interaction force and apply the contact loss criterion. This iterative procedure is schematised in Algorithm 1. It keeps going until the dropper slackening state $\mathscr{D}$ and the contact loss state $\mathscr{C}$ are identical in two consecutive 
iterations. When this happens, we can also move to the next time step $t+1$.

The main feature of this classical approach is that a different system of equations of the global problem size is solved several times in each time step, which requires a significant computational effort. Another disadvantage of this method emerges when changes in the pantograph model are studied, because it entails a different resolution of the whole dynamic interaction problem for every change introduced in the pantograph model.

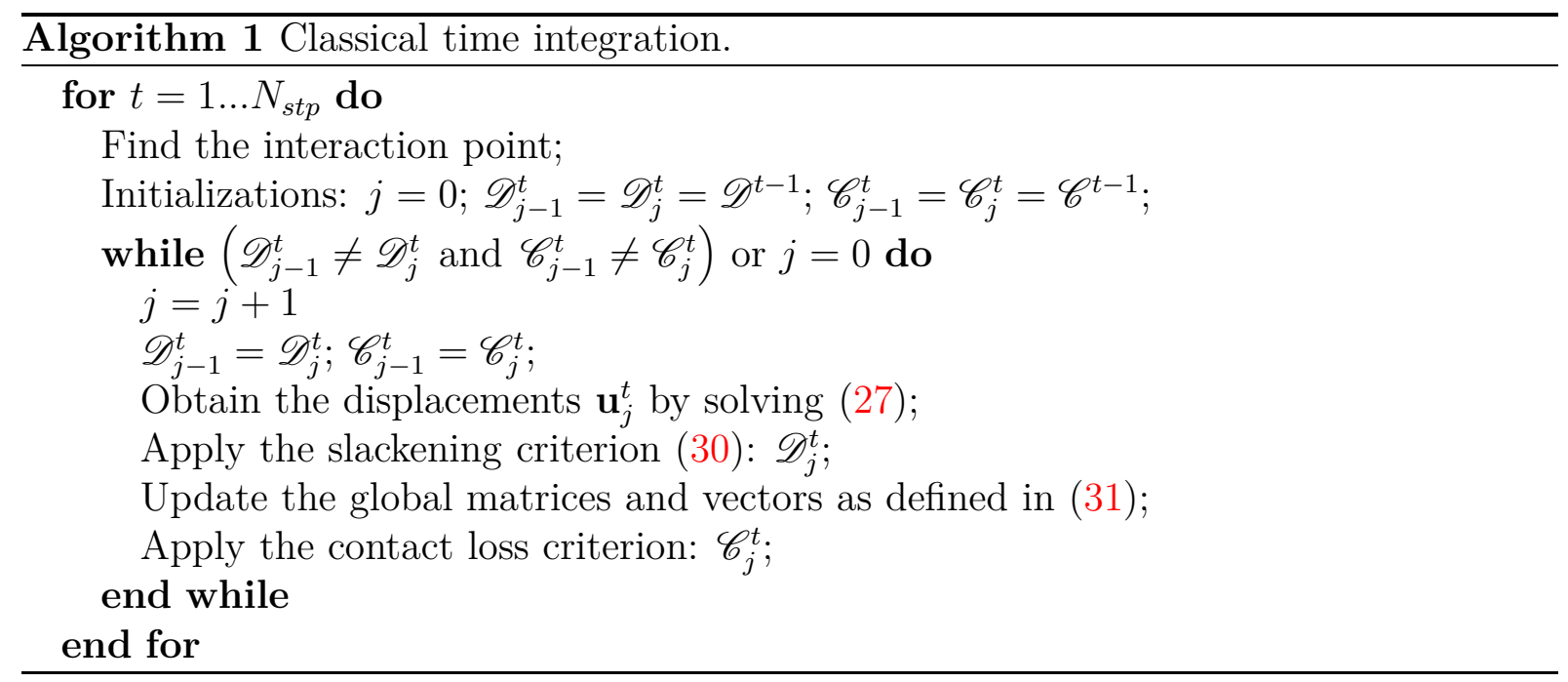

\subsection{Modified time integration procedures}

As mentioned above, the main drawback of the classical approach is the reassembling the global matrix at each time step, which makes this procedure inappropriate in practice, due to the high computational cost required. Some ideas have been proposed to circumvent this issue in [17] and exploited in [30], considerably improving the computational cost. Specifically, the non-linearities of the system are moved to the right hand side of the dynamic equilibrium equation, and are therefore treated as non-linear forces. In this way, the global time-step matrix $\mathbf{A}_{j}^{t}$ does not vary in time, keeping the analysis linear. Hence, it can be factorised only once, reducing the computational effort. In practice, this modification of the classical approach can be carried out according to the following two procedures:

- Method 1: The global matrix of the system does not include the stiffness and 
damping of all dropper elements. They are fully treated as non-linear forces instead.

- Method 2: The droppers' stiffness and damping are fully accounted for in the global matrix of the system as in [30]. In this case, the non-linear force term added to the right hand side of the dynamic equation compensates for the slackened droppers.

Despite the clear advantages of these approaches over the classical method, in order to account for the non-linear behaviour of the droppers and the pantograph loss of contact, the iterative procedure requires solving a global size system several times in each time step in which the nodal displacements are still the unknowns of the problem.

\subsection{Offline/Online time integration methodology}

Starting from the ideas of the modified Method 2, we try to concentrate as much computational effort as possible on the Offline stage, leaving the minimum number of calculations to the so-called Online stage, in which the time integration itself takes place. Another important feature of the proposed methodology is that both the pantograph and the catenary are treated as independent systems, which makes it easier to deal with different pantograph models, or even a real system, as in HIL simulations.

In the Offline phase of the algorithm, the catenary is treated as a fully linear system in which the droppers are not able to slacken. Several single time step problems are solved and stored at this stage. In the Online calculations, the superposition principle is applied and both the initial conditions of movement and all the non-linearities of the system are considered, i.e. dropper slackening and pantograph contact loss. Using the information calculated in the Offline stage, these non-linearities are accounted for iteratively by solving a very small system of equations in which the unknowns are no longer the nodal displacements, but the slackening compensating forces and the pantograph-catenary interaction force. This small set of unknowns is responsible for reducing the computational cost of the solution method. 


\subsubsection{Offline stage}

All the calculations which take place before the time integration loop are called Offline stage. In this phase we solve several sub-problems whose solution will be used afterwards in the Online computations. All these sub-problems are aimed at obtaining the single time-step forced response of the system under a unitary external load and null initial conditions. These unitary external forces are applied at the interaction point and at the dropper ends, as shown in Fig. 6.

As the pantograph moves forward throughout a dynamic simulation, the contact point changes in each time step. The goal of the first sub-problem in this stage is to calculate the response of the catenary system considering a unitary vertical force pushing upwards at each point at which the pantograph interacts with the contact wire (dashed arrows in Fig. 6). We have to solve as many linear problems of the form

$$
\mathbf{A}_{c a t} \mathbf{u}_{i, c a t}^{*, t}=\mathbf{F}_{i, c a t}^{*, t}
$$

as total time steps $N_{s t p}$ in order to find the displacements $\mathbf{u}_{i, c a t}^{*, t}$. Recalling the idea introduced in [17], it is important to underline that the matrix of this linear system

$$
\mathbf{A}_{c a t}=\left[(1+\alpha)\left[\mathbf{K}_{c a t}+b_{4} \mathbf{C}_{c a t}\right]+b_{1} \mathbf{M}_{c a t}\right]
$$

does not change in each time step $t$ and therefore for any load position. Hence, it can be factorised as $\mathbf{A}_{c a t}=\mathbf{L}_{c a t} \mathbf{U}_{c a t}$, where $\mathbf{L}_{c a t}$ and $\mathbf{U}_{c a t}$ are lower and upper triangular matrices, respectively. Applying the Cuthill-McKee reordering algorithm [31] to matrix $\mathbf{A}_{c a t}$, it is possible to obtain very sparse matrices $\mathbf{L}_{c a t}$ and $\mathbf{U}_{c a t}$ which will reduce the computational cost in the Online calculations.

The other necessary catenary responses, which also will be used in the next stage, are obtained considering the unitary external forces acting on the ends of each of the $N_{d}$ droppers of the catenary (solid arrows in Fig. 6). These external forces are aligned with droppers. The displacement field $\mathbf{u}_{d, c a t}^{*}$ is obtained as the solution of the one time-step problem:

$$
\mathbf{A}_{c a t} \mathbf{u}_{d, c a t}^{*}=\mathbf{F}_{d, c a t}^{*}
$$

in which now $d=1, \ldots, N_{d}$. Note that $N_{s t p}+N_{d}$ linear problems for the catenary must be 
solved, but as mentioned above they are performed Offline and only once.

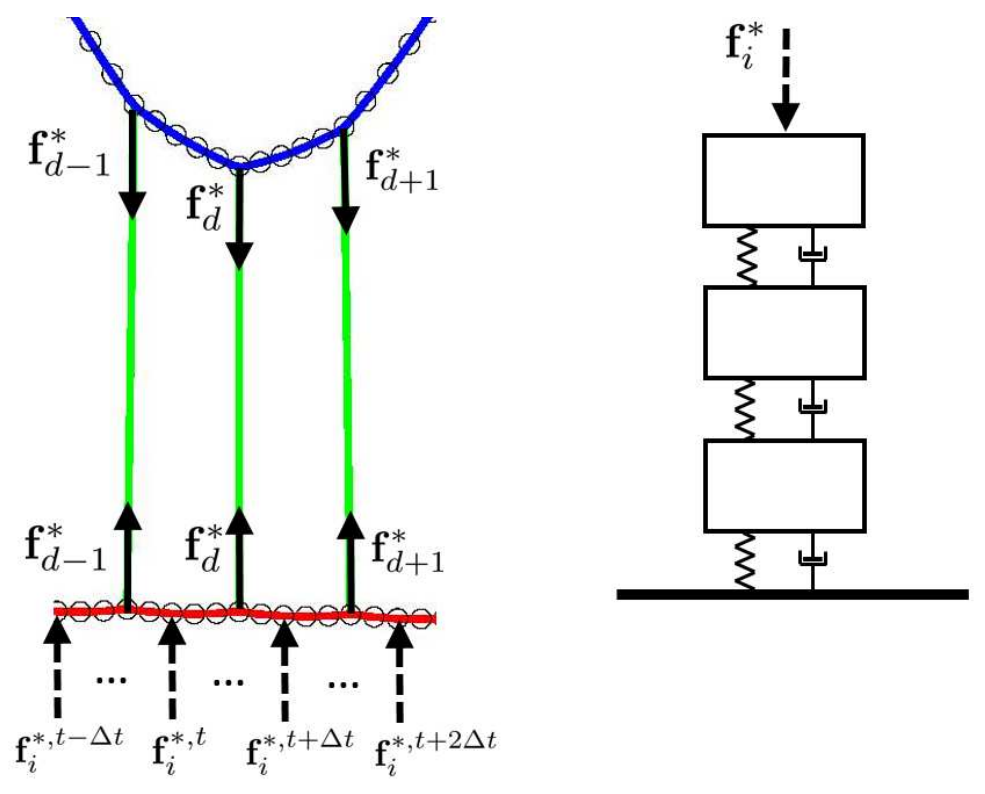

Figure 6: Unitary external forces applied in the Offline stage.

Finally, we only need the pantograph response under a unitary force pushing downwards on the top mass where the interaction with the contact wire takes place. The dynamic response of one time step provided by the HHT integrator is $\mathbf{w}_{i, p a n}^{*}$, which does not depend on the time step. The only problem to solve related with the pantograph is:

$$
\mathbf{A}_{\text {pan }} \mathbf{w}_{i, p a n}^{*}=\mathbf{F}_{i, p a n}^{*}
$$

where the matrix

$$
\mathbf{A}_{\text {pan }}=\left[(1+\alpha)\left[\mathbf{K}_{\text {pan }}+b_{4} \mathbf{C}_{p a n}\right]+b_{1} \mathbf{M}_{\text {pan }}\right]
$$

is also constant regardless the time step that is being solved.

The velocities and accelerations of all the sub-problems described above are also computed following the rules of the HHT time integrator.

\subsubsection{Online stage}

In this stage the time integration is carried out taking into account the non-linearities introduced by dropper slackening and pantograph contact loss. In order to obtain the 
formulation of the proposed approach we start from Eq. (27). Proceeding as in the modified approaches introduced in Section 4.2, the terms involving the interaction, as well as the corrections forces for the slackened droppers, are moved to the right hand side of the dynamic equation. It results in:

$$
\begin{aligned}
& {\left[(1+\alpha)\left[\left(\begin{array}{cc}
\mathbf{K}_{c a t} & \mathbf{0} \\
\mathbf{0} & \mathbf{K}_{\text {pan }}
\end{array}\right)+b_{4}\left(\begin{array}{cc}
\mathbf{C}_{c a t} & \mathbf{0} \\
\mathbf{0} & \mathbf{C}_{p a n}
\end{array}\right)\right]+b_{1} \mathbf{M}\right] \mathbf{u}^{t}=\mathbf{F}_{I C}^{t}+\mathbf{F}_{\text {pan }}-} \\
& \alpha\left(-\mathbf{S}_{i}^{t-1}-\mathbf{K}_{i}^{t-1} \mathbf{u}^{t-1}+\sum_{d}^{N_{s d}} \mathbf{S}_{d}^{t-1}+\mathbf{K}_{d}^{t-1} \mathbf{u}^{t-1}+\mathbf{C}_{d}^{t-1} \dot{\mathbf{u}}^{t-1}\right)+ \\
& (1+\alpha)\left(-\mathbf{S}_{i}^{t}-\mathbf{K}_{i}^{t} \mathbf{u}^{t}+\sum_{d}^{N_{s d}} \mathbf{S}_{d}^{t}+\mathbf{K}_{d}^{t} \mathbf{u}^{t}+\mathbf{C}_{d}^{t} \dot{\mathbf{u}}^{t}\right)
\end{aligned}
$$

where, by the use of the hypotheses assumed in the HHT algorithm, the velocity $\dot{\mathbf{u}}^{t}=$ $b_{4}\left(\mathbf{u}^{t}-\mathbf{u}^{t-1}\right)+b_{5} \dot{\mathbf{u}}^{t-1}+b_{6} \ddot{\mathbf{u}}^{t-1}$. It is also important to mention that in this rearrangement there is no coupling between pantograph and catenary degrees of freedom in the system of equations. By grouping terms, the previous equation can be rearranged as:

$$
\mathbf{A} \mathbf{u}^{t}=\mathbf{F}_{k n}^{t}+(1+\alpha)\left(\mathbf{F}_{i}^{t}+\sum_{d}^{N_{s d}} \mathbf{F}_{d}^{t}\right)
$$

where

$$
\begin{gathered}
\mathbf{F}_{k n}^{t}=\mathbf{F}_{I C}^{t}+\mathbf{F}_{p a n}-\alpha\left(-\mathbf{S}_{i}^{t-1}-\mathbf{K}_{i}^{t-1} \mathbf{u}^{t-1}+\sum_{d}^{N_{s d}}\left(\mathbf{S}_{d}^{t-1}+\mathbf{K}_{d}^{t-1} \mathbf{u}^{t-1}+\mathbf{C}_{d}^{t-1} \dot{\mathbf{u}}^{t-1}\right)\right) \\
\mathbf{F}_{i}^{t}=-\mathbf{S}_{i}^{t}-\mathbf{K}_{i}^{t} \mathbf{u}^{t} \\
\mathbf{F}_{d}^{t}=\mathbf{S}_{d}^{t}+\mathbf{K}_{d}^{t} \mathbf{u}^{t}+\mathbf{C}_{d}^{t} \dot{\mathbf{u}}^{t}
\end{gathered}
$$

In this expression, $\mathbf{F}_{k n}^{t}$ groups all the known forces: those coming from the initial conditions, the constant uplift force and the forces arising from the previous time step. Thus, the unknown forces at time step $t$ are the interaction force $\mathbf{F}_{i}^{t}$ and the slackened dropper correction forces $\mathbf{F}_{d}^{t}$, since they depend on the sought displacements $\mathbf{u}^{t}$.

The total displacement $\mathbf{u}^{t}$ in Eq. (38) is the response of two linear systems (catenary and pantograph) subjected to the three different actions defined in Eq. (39), two of which depend on $\mathbf{u}^{t}$ itself. Hence, the total response of the system can be computed as the sum of the responses caused by these forces acting separately. Denoting as $\mathbf{u}_{F_{k n}}^{t}, \mathbf{u}_{F_{i}}^{t}$ and $\mathbf{u}_{F_{d}}^{t}$ 
the displacement produced respectively by the forces $\mathbf{F}_{k n}^{t}, \mathbf{F}_{i}^{t}$ and $\mathbf{F}_{d}^{t}$, the total response of the system is computed as:

$$
\mathbf{u}^{t}=\mathbf{u}_{F_{k n}}^{t}+(1+\alpha)\left(\mathbf{u}_{F_{i}}^{t}+\sum_{d}^{N_{s d}} \mathbf{u}_{F_{d}}^{t}\right)
$$

or equivalently, taking benefit from the responses under unitary forces calculated in the Offline stage, and explicitly splitting the system in terms of catenary and pantograph:

$$
\left(\begin{array}{c}
\mathbf{u}_{c a t}^{t} \\
\mathbf{w}_{\text {pan }}^{t}
\end{array}\right)=\left(\begin{array}{c}
\mathbf{u}_{F_{k n}, c a t}^{t} \\
\mathbf{w}_{F_{k n}, p a n}^{t}
\end{array}\right)+(1+\alpha)\left(f_{i}^{t}\left(\begin{array}{c}
\mathbf{u}_{i, c a t}^{* t} \\
\mathbf{w}_{i, p a n}^{*}
\end{array}\right)+\sum_{d}^{N_{s d}} f_{d}^{t}\left(\begin{array}{c}
\mathbf{u}_{d, c a t}^{*} \\
\mathbf{0}
\end{array}\right)\right)
$$

Now, the magnitude of the interaction force $f_{i}^{t}$ and the internal forces of slackened droppers $f_{d}^{t}$ for $d=1, \ldots, N_{s d}$ at instant $t$, are the set of unknowns of the problem. $\mathbf{u}_{F_{k n}, c a t}^{t}$ and $\mathbf{w}_{F_{k n}, \text { pan }}^{t}$ are easy to compute because you only must solve the systems $\mathbf{A}_{c a t} \mathbf{u}_{F_{k n}, c a t}^{t}=\mathbf{F}_{k n, c a t}^{t}$ and $\mathbf{A}_{p a n} \mathbf{w}_{F_{k n}, p a n}^{t}=\mathbf{F}_{k n, p a n}^{t}$. Although the first of these systems of equations can be quite large, we remember that the matrix of the system has been factorized in the Offline stage into two sparse triangular matrices. Thus, solving the whole system consists of applying forward and backward solvers which are computationally very efficient. Besides, this global size system need only be solved once at each time step, unlike the Methods 1 and 2 defined in Section 4.2, which require several solutions of systems of this size in every time step.

To deal with the non-linearities, we define the vector $\mathbf{f}=\left[f_{i}^{t} f_{1}^{t} \cdots f_{N_{s d}}^{t}\right]^{T}$, which contains all the unknowns of Eq. (41). Note that although they are forces, only their magnitude is unknown, since their direction has been previously established. It is also important to emphasize that the number of unknowns is significantly smaller than the total number of degrees of freedom of the system. In order to find these unknowns, due to the nonlinearities introduced by the dropper slackening and the pantograph contact loss, we must set and solve iteratively a system of equations of variable size because we do not know which droppers are slackened.

The first equation of such a system comes from the force balance at the interaction point. Looking at the second expression of Eq. (39) (or equivalently Eq. (22)) and introducing the solution in terms of Eq. (41), the external force at the contact point that must be 
applied is:

$$
\begin{gathered}
f_{i}^{t}=k_{h}\left(z_{r e f}+w_{p a n}^{t}-z_{0, c w}^{t}-w_{c w}^{t}\right)=k_{h}\left(z_{r e f}+w_{F_{k n}, p a n}^{t}+(1+\alpha) f_{i}^{t} w_{i, p a n}^{*}-z_{0, c w}^{t}-\right. \\
\left.w_{F_{k n}, c w}^{t}-(1+\alpha)\left(f_{i}^{t} w_{i, c w}^{* t}+\sum_{d}^{N_{s d}} f_{d}^{t} w_{d, c w}^{*}\right)\right)
\end{gathered}
$$

The remaining $N_{s d}$ equations of the system are derived from the third expression of Eq. (39) particularized on every slackened dropper $d$. By doing this we obtain the compensating forces on both nodes of the slackened dropper element. In order to obtain a scalar equation, we select only the forces on the node $n$ and project them in the direction $\mathbf{n}_{d}$. Finally, introducing Eq. (41) again, and its velocity counterpart, we obtain:

$$
\begin{aligned}
f_{d}^{t}= & \mathbf{n}_{d} \cdot\left(\mathbf{s}_{d}+\left[\mathbf{k}_{d} \mathbf{u}_{d}^{t}+\mathbf{c}_{d} \dot{\mathbf{u}}_{d}^{t}\right]_{n}\right)= \\
& \mathbf{n}_{d} \cdot\left(\mathbf{s}_{d}+\left[\mathbf{k}_{d}\left(\mathbf{u}_{F_{k n}, d}^{t}+(1+\alpha)\left(f_{i}^{t} \mathbf{u}_{i, d}^{* t}+\sum_{m}^{N_{s d}} f_{m}^{t} \mathbf{u}_{m d}^{*}\right)\right)+\right.\right. \\
& \left.\left.\mathbf{c}_{d}\left(\dot{\mathbf{u}}_{F_{k n}, d}^{t}+(1+\alpha)\left(f_{i}^{t} \dot{\mathbf{u}}_{i, d}^{* t}+\sum_{m}^{N_{s d}} f_{m}^{t} \dot{\mathbf{u}}_{m d}^{*}\right)\right)\right]_{n}\right)
\end{aligned}
$$

Rearranging Eqs. (42) and (43) in a matrix form leads to:

$$
\left(\begin{array}{ll}
a & \mathbf{b} \\
\mathbf{c} & \mathbf{D}
\end{array}\right) \mathbf{f}=\left[\begin{array}{l}
e \\
\mathbf{g}
\end{array}\right]
$$

Recalling that the index $d=1, \ldots, N_{s d}$, the matrix of the system is defined by

$$
a=\frac{1}{k_{h}}-(1+\alpha)\left(w_{i, p a n}^{*}-w_{i, c w}^{* t}\right)
$$

the row vector $\mathbf{b}$ with the entries

$$
b_{d}=(1+\alpha) w_{d, c w}^{*},
$$

the column vector $\mathbf{c}$ such that

$$
c_{d}=-(1+\alpha) \mathbf{n}_{d} \cdot\left[\mathbf{k}_{d} \mathbf{u}_{i, d}^{* t}+\mathbf{c}_{d} \dot{\mathbf{u}}_{i, d}^{* t}\right]_{n},
$$


and the squared matrix $\mathbf{D}=\mathbf{I}_{N_{s d}}-\mathbf{G}$, being $\mathbf{I}_{N_{s d}}$ the identity matrix of size $N_{s d}$ and $\mathbf{G}$ a matrix whose entries are

$$
g_{d d}=-(1+\alpha) \mathbf{n}_{d} \cdot\left[\mathbf{k}_{d} \mathbf{u}_{d d}^{*}+\mathbf{c}_{d} \dot{\mathbf{u}}_{d d}^{*}\right]_{n}
$$

The right hand side vector is composed of

$$
e=z_{r e f}-z_{0, c w}^{t}+w_{F_{k n}, p a n}^{t}-w_{F_{k n}, c w}^{t}
$$

and the column vector $\mathbf{g}$ with the components

$$
g_{d}=\mathbf{n}_{d} \cdot\left(\mathbf{s}_{d}+\left[\mathbf{k}_{d} \mathbf{u}_{F_{k n}, d}^{t}+\mathbf{c}_{d} \dot{\mathbf{u}}_{F_{k n}, d}^{t}\right]_{n}\right)
$$

After solving the system defined in (44), the fulfilment of the slackening (30) and the contact loss criteria are checked out. For the next iteration, only the droppers which slacken will take part in the linear system of equations (44). This iterative procedure is summarized in Algorithm 2. As in the classical approach, it lasts until in two consecutive iterations the state of all droppers $\mathscr{D}$ and the contact loss state $\mathscr{C}$ are equal, which is always achieved with only 3 or at most 4 iterations in the numerical examples that we have solved. When the iterative procedure ends, one can move to the next time step of the time integration scheme.

With this proposed method the pantograph and the catenary are absolutely independent, which allows us to use the proposed method with a real pantograph in a HIL simulation. In such a case, the contact force would be an input at every time step. The computational cost of this methodology is much smaller (even achieving real-time response) than the one required in the classical approach and its modified versions. For every time step we only perform a unique global backward and forward resolution with very sparse triangular matrices, and a linear system of equations of the small size $N_{s d}+1$ must be set and solved few times, instead of solving the global size system several times at each time step, as in the classical approaches. 


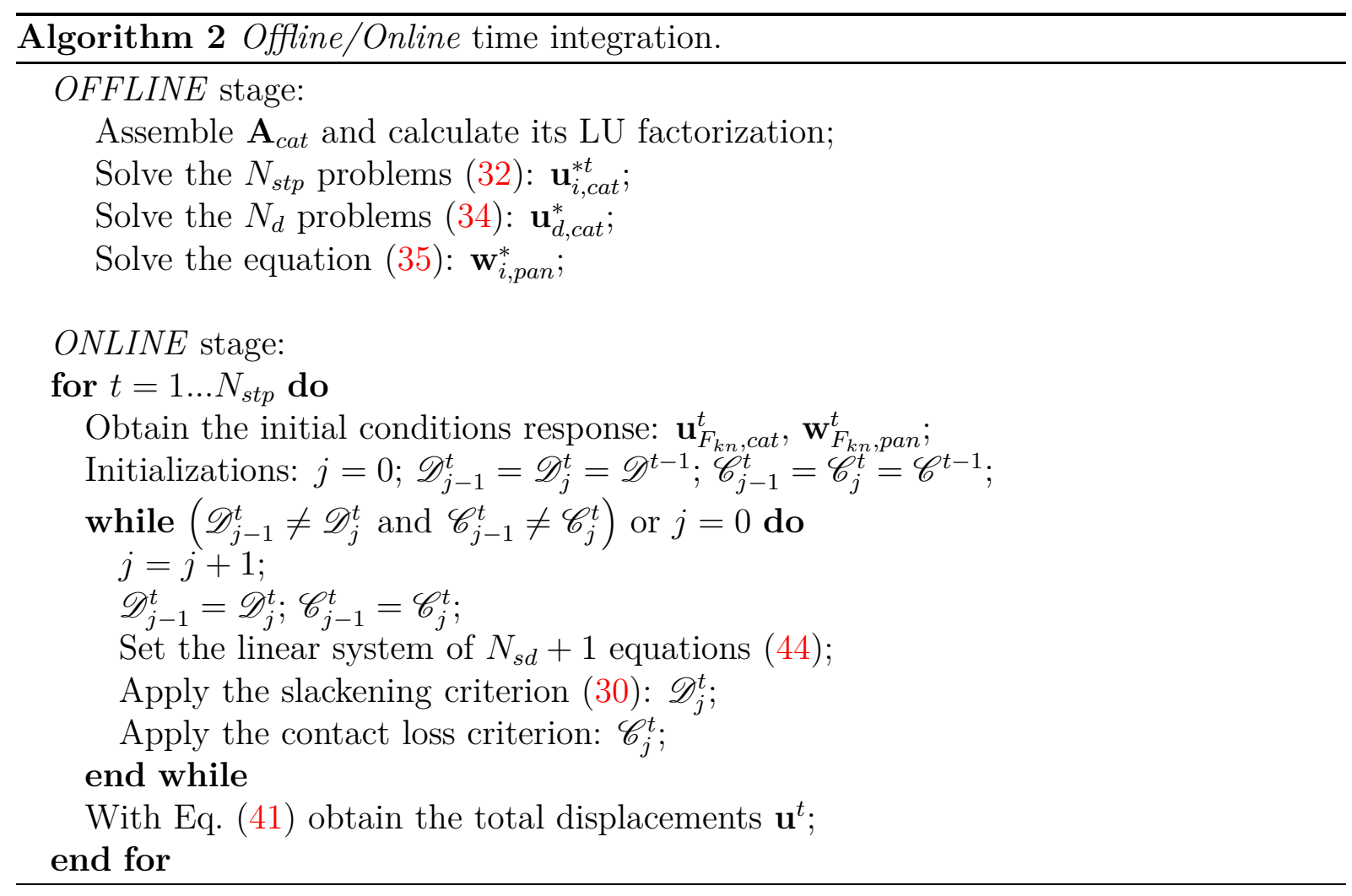

\section{Numerical examples}

The numerical examples given in this section are intended to verify the simplifying hypothesis we assumed for the formulation of the dynamic problem and also, to demonstrate the significant reduction in computational cost offered by the proposed Offline/Online method, as compared with the classical approaches.

\subsection{Hypothesis verification}

In the beginning of Section 4, we applied two important simplifications. The first concerns the linearisation of the dynamic equations with respect to the static equilibrium position of the catenary cables. With this assumption we got rid of the geometrical non-linearity introduced by the strain measure. The second simplification is based on the small deformation undergone by the droppers in the static equilibrium configuration. This leads to an elemental stiffness matrix which is independent of the absolute position in which the 


\begin{tabular}{|c|c|c|c|c|}
\hline Component & $E(\mathrm{MPa})$ & $\rho\left(\mathrm{kg} / \mathrm{m}^{3}\right)$ & $A\left(\mathrm{~mm}^{2}\right)$ & $I\left(\mathrm{~mm}^{4}\right)$ \\
\hline \hline Messenger wire & 9114 & $1.1 \cdot 10^{11}$ & 94.8 & 1237.2 \\
\hline Contact wire & 9160 & $1.1 \cdot 10^{11}$ & 150 & 2170 \\
\hline Droppers & 9114 & $1.1 \cdot 10^{11}$ & 10 & 0 \\
\hline
\end{tabular}

Table 1: Material and geometrical properties of the catenary components.

system has been linearised.

To check whether or not these assumptions affect the solution, we are going to simulate the same problem formulated with the non-linear behaviour and the two versions of the linearised dynamic equations. For the simulations we use the catenary model proposed in [3], whose geometry is depicted in Fig. 7. This is a 3D catenary with a stagger of $20 \mathrm{~cm}$ from the centre line of the track. It is composed of 10 spans $55 \mathrm{~m}$ long and 9 droppers each. The material properties of the different components are listed in Table 1. A proportional Rayleigh damping model is defined by the constants $\alpha_{r}=0.0125$ and $\beta_{r}=0.0001$.

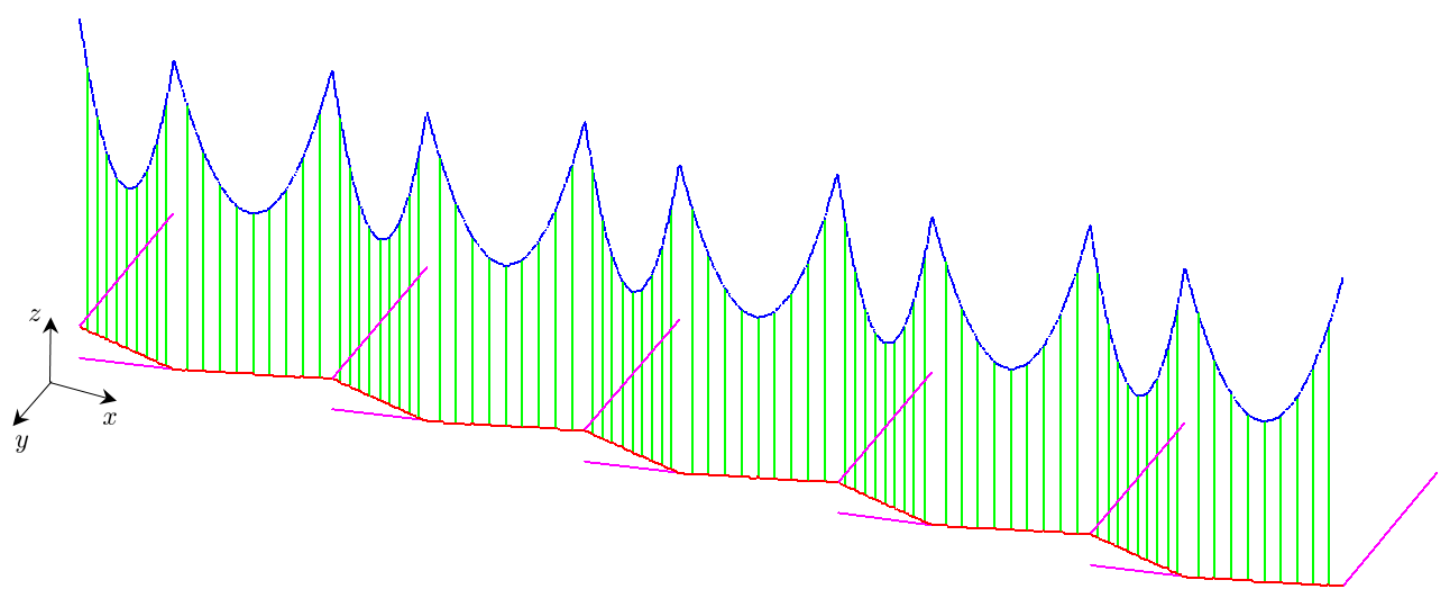

Figure 7: Catenary geometry used for the hypothesis validation.

The parameters used for the lumped mass pantograph model are shown in Table 2. The applied uplift force is $F_{p a n}=180 \mathrm{~N}$. A time step of $\Delta t=0.001 \mathrm{~s}$ is used for the dynamic simulation. The pantograph moves at $270 \mathrm{~km} / \mathrm{h}$ and the HHT parameters are set to $\alpha=-0.05, \beta=0.2756$ and $\gamma=0.55$. The penalty stiffness assigned for the interaction model is $k_{h}=50000 \mathrm{~N} / \mathrm{m}$.

The results shown in the figures below are focused on the contact force at the two central spans in order to minimize unwanted contributions from boundary effects. This magnitude 


\begin{tabular}{|c|c|c|c|}
\hline dof & $m(\mathrm{~kg})$ & $c(\mathrm{Ns} / \mathrm{m})$ & $k(\mathrm{~N} / \mathrm{m})$ \\
\hline \hline 1 & 6.6 & 0 & 7000 \\
\hline 2 & 5.8 & 0 & 14100 \\
\hline 3 & 5.8 & 70 & 80 \\
\hline
\end{tabular}

Table 2: Pantograph model parameters.

is considered a good representative output of the dynamic simulation. Fig. 8 shows the interaction force obtained from two simulations, one using the non-linear formulation (solid line) and the other with the linearised formulation (dashed line). At first glance no difference is observed, but plotting the relative error between the two curves shows that it does not exceed $3.4 \%$, confirming the validity of the linearisation hypothesis.
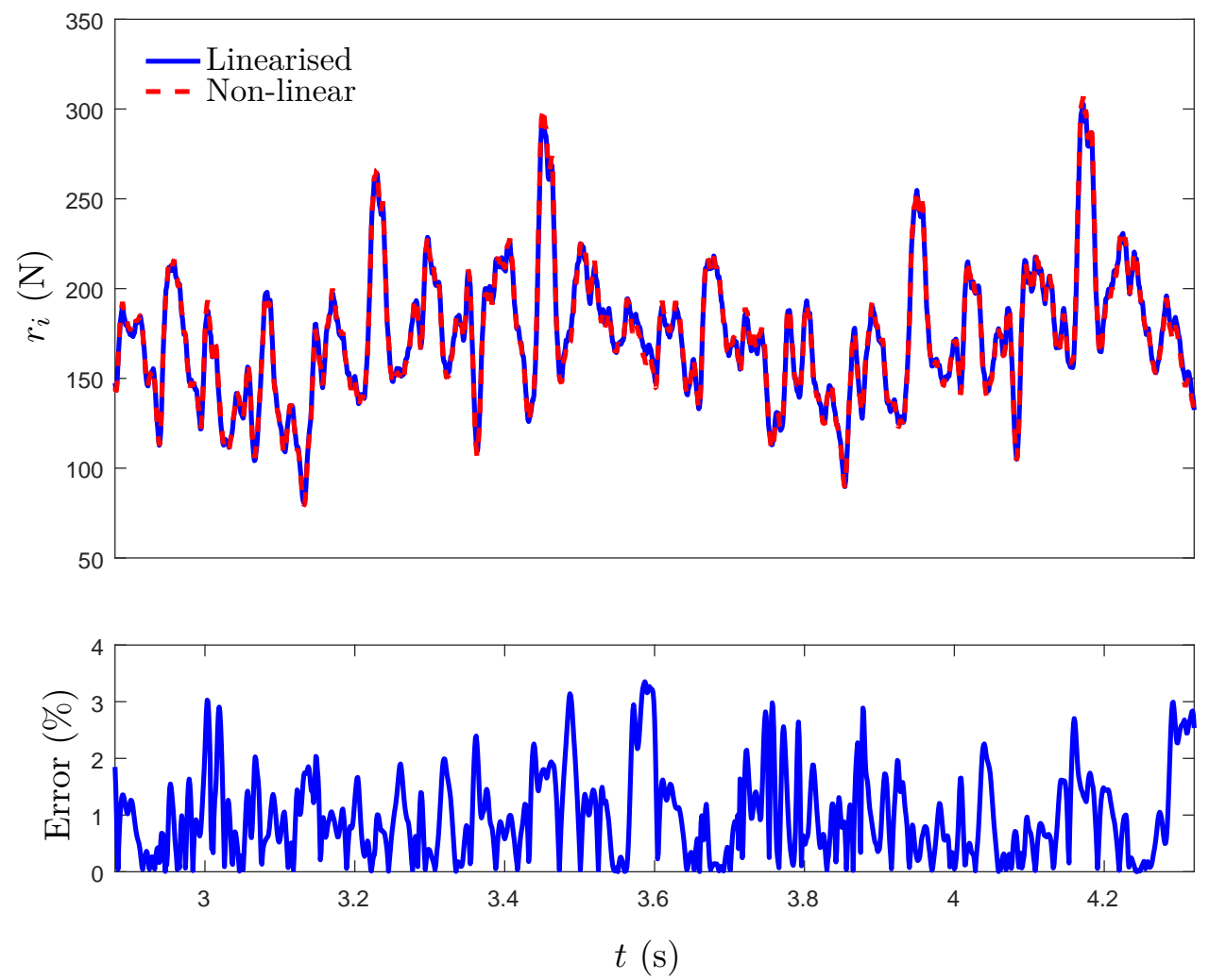

Figure 8: Interaction force from the linearised (solid blue line) and the nonlinear (dashed red line) formulation. Relative error between them.

As regards the second assumption, Fig. 9 shows the interaction force obtained considering the full dropper stiffness matrix (solid line) or its simplified version (dashed line). Again, 
the differences between the two simulations are negligible, with a mean relative error around $1 \%$.

Considering the two simplifications together, the mean of the relative error in the contact force between the linearised formulation using the simplified dropper stiffness matrix and the non-linear problem is less than 1.6\%. Hence, we can conclude that using the linearised formulation along with the simplified dropper stiffness matrix (18) does not significantly affect the results.
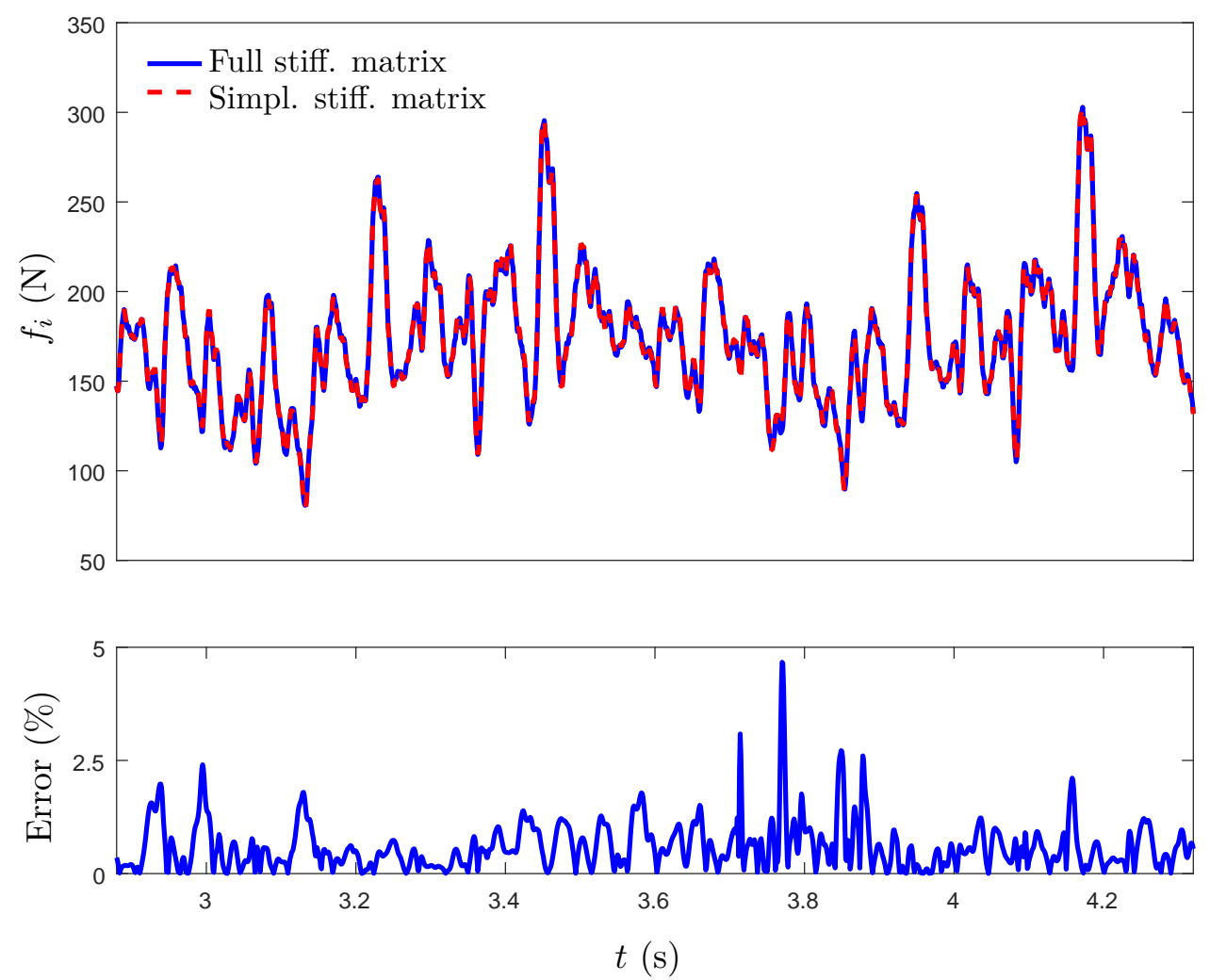

Figure 9: Interaction force using the full dropper stiffness matrix (solid blue line) or the simplified one (dashed red line). Relative error between them.

After verifying the simplifications introduced into the formulation, we should now investigate other changes that may save computational cost. Specifically, we are going to look for the largest time step $\Delta t$ in the HHT algorithm that provides accurate results.

For this purpose, the interaction force obtained with three different time steps is compared 
in Fig. 10. The use of $\Delta t=0.002 \mathrm{~s}$ (dashed red curve), produces quite accurate results when compared with the ones (solid blue curve) obtained with $\Delta t=0.001$ s. However, if $\Delta t=0.005 \mathrm{~s}$ the solution does not match with the reference interaction force at all. Hence, in the light of these results, we can select the time step of $2 \mathrm{~ms}$.

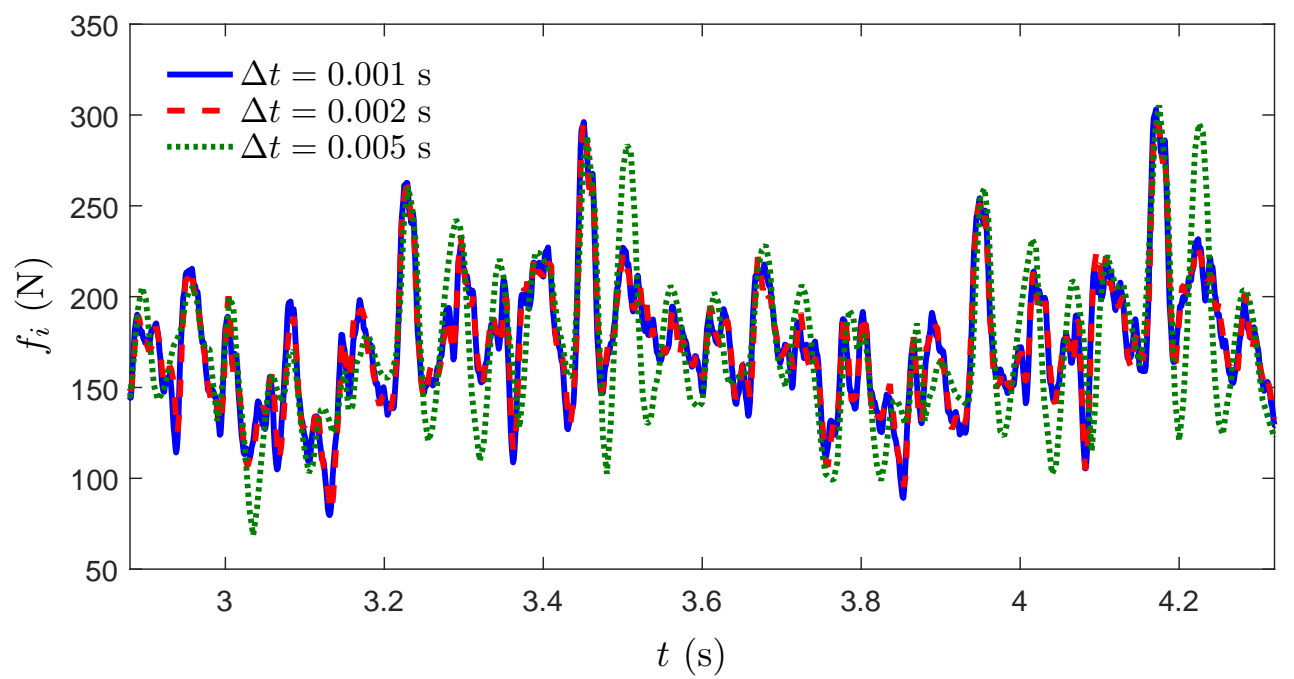

Figure 10: Interaction force using different time steps.

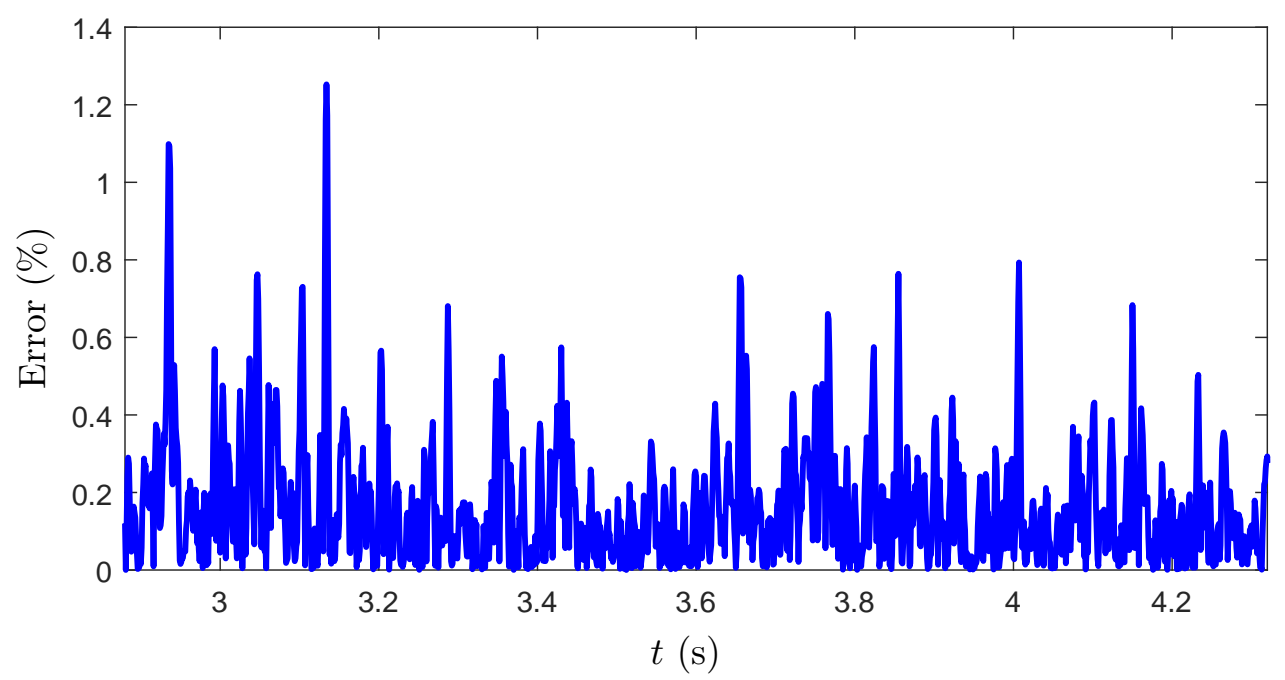

Figure 11: Relative error between the solution obtained with $\alpha=0$ and $\alpha=-0.05$.

Another aspect to analyse is the value of the constant $\alpha$ in the HHT method. Fig. 11 represents the relative error of the interaction force obtained from $\alpha=0$ (equivalent to the Newmark method) and $\alpha=-0.05$. As can be seen, this error does not exceed $1 \%$ in 
most of the interest interval. This means the Newmark method can be used at a lower computational cost because we do not need to include in Eq. (37) the terms evaluated in the previous time step $t-1$.

\subsection{Computation time study}

The classical approach and the proposed Offline/Online methodology come from the same dynamic equilibrium equation. This means that there are no differences in the time history of the interaction force, obtained by both methods. This is why in this section we only compare the computational cost of the commonly used classical approaches and the proposed Offline/Online methodology.

To carry out this comparison, four different catenary models are studied. Three of them are 3D models with 5 (Cat.1), 10 (Cat.2) and 18 (Cat.3) spans respectively, and the other one is a $2 \mathrm{D}$ model $1 \mathrm{~km}$ in length and with 18 spans (Cat.4).

As mentioned previously, the displacements, velocities and accelerations calculated in the Offline stage must be stored to be used in the succeeding Online phase. This requires a certain amount of RAM memory available in the computer in order to avoid swapping data on the hard disk, which would slowdown the computations. For Cat.3, the biggest example studied here, approximately $2.5 \mathrm{~GB}$ of RAM memory is large enough to store all the results.

In Table 3 we compare the computational cost required to perform the simulations with these four catenary models. The first two rows show the number of degrees of freedom of each catenary model and the total time simulated, respectively. All the simulations are carried out with a time step $\Delta t=0.002 \mathrm{~s}$, and the rest of the time integration parameters are equal, as in the previous section. The code was implemented in MATLAB ${ }^{\circledR}$ and launched in an Intel ${ }^{\circledR}$ Core i7-6700 CPU.

The computational time required for the classical approach time integration, the two modified methods, the Offline stage of the new approach and also the Online phase are displayed using both HHT and Newmark methods.

The remarkably high computational cost required for the modified Method 1 is about four 


\begin{tabular}{|c|c|c|c|c|}
\hline & Cat.1 & Cat.2 & Cat.3 & Cat.4 \\
\hline \hline dofs & 5996 & 11986 & 21570 & 14385 \\
\hline Simulated time (s) & 3 & 5 & 10 & 10 \\
\hline \hline
\end{tabular}

\section{HHT}

\begin{tabular}{|c|c|c|c|c|}
\hline Classical (s) & 56.05 & 205.38 & 802.80 & 412.46 \\
\hline Modified 1 (s) & 156.76 & 676.1 & 2575.2 & 1405.7 \\
\hline Modified 2 (s) & 20.98 & 90.58 & 325.47 & 187.35 \\
\hline Offline stage (s) & 0.76 & 2.39 & 9.83 & 5.28 \\
\hline Online stage (s) & 1.74 & 5.22 & 19.27 & 10.86 \\
\hline \hline
\end{tabular}

Newmark

\begin{tabular}{|c|c|c|c|c|}
\hline Classical (s) & 52.69 & 218.74 & 785.10 & 417.60 \\
\hline Modified 1 (s) & 91.99 & 323.89 & 1270 & 757.8 \\
\hline Modified 2 (s) & 14.93 & 50.87 & 201.24 & 111.64 \\
\hline Offline stage (s) & 0.79 & 2.50 & 9.64 & 5.19 \\
\hline Online stage (s) & 1.37 & 4.48 & 15.75 & 9.10 \\
\hline \hline
\end{tabular}

Table 3: Computational time comparison.

times more than the classical algorithm. This is because the matrix of the system does not include any information of droppers, leading to convergence problems in the iterative procedure, which needs a large number of iterations to obtain accurate solutions. Hence, although the system matrix has been factorised, there is no improvement in computational cost, due to the large number of times the global size system needs to be solved.

However, there is a noticeable time gain with the use of the modified Method 2. In this case, the time integration can be solved more than three times faster than with the classic scheme. In this procedure, apart from taking advantage of having a factorised matrix, no more than ten iterations are usually needed to achieve convergence with a good degree of accuracy.

The good performance of the proposed Offline/Online method can be clearly seen in Table 3. The same simulation with exactly the same accuracy is carried out around 25 times faster than with the classic approach. This total gain factor is slightly increased for large catenary models, which require more computational effort. Focusing only on the time consumed by the Online stage, in most cases it comes close to (light green boxes), or is even less than the simulated time (green boxes), which allows real-time responses for HIL simulations. These low computational costs are due to the way we treat the nonlinearities; for the four catenaries solved, they account for solving iteratively a system of 
maximum size of 6 equations, with only 4 iterations at most.

It is very clear that, regardless of the catenary model, the proposed approach is highly suitable for optimization purposes, when a large number of simulations must be performed.

\section{Conclusions}

This paper deals with the numerical simulation of pantograph-catenary dynamic interaction. The catenary is modelled by FE according to the absolute nodal coordinate formulation, while the pantograph is treated as a lumped mass system. The major difficulty in the simulations lies in the nonlinearities introduced by dropper slackening and the possible loss of contact of the pantograph. With a classical approach, a linear system of equations of the overall size of the problem must be solved several times at each time step in order to account for these nonlinearities. This has a considerable computational cost and makes the approach useless for optimization purposes, in which many simulations are required.

Certain modifications are intended to avoid reassembling the matrix each time step by dealing with the nonlinearities as forces keeping the global matrix constant in time. Starting from these modified formulations, we developed a new strategy that notably reduces the computational effort in each simulation without any loss of accuracy. The proposed procedure is based on two stages: the Offline phase, performed only once, in which we solve several single time-step problems, applying unitary forces. This is followed by the Online stage in which we account for the initial conditions and deal with the nonlinearities by only solving a very small system of equations whose unknowns are the interaction force and the slackened dropper correction forces.

The Offline/Online method results in a highly computational-cost-saving approach, making it a very suitable tool for optimizing the catenary and pantograph models. Furthermore, it has been shown to be capable of computing the real-time response of the catenary unlike any other previous method in which there were no losses in accuracy. This means that the Offline/Online approach can be used to implement HIL simulations, in which a pantograph model is replaced by a real system. 


\section{Appendix A. The HHT time integration method}

The Hilber-Hughes-Taylor (HHT) method [29] is an implicit time integration scheme widely used in structural dynamics. Given the linear system of equations of motion:

$$
\mathbf{M u}+\mathbf{C} \dot{\mathbf{u}}+\mathbf{K u}=\mathbf{F}
$$

the HHT method is based on the Newmark hypotheses:

$$
\begin{aligned}
& \mathbf{u}^{t+1}=\mathbf{u}^{t}+\Delta t \dot{\mathbf{u}}^{t}+\frac{\Delta t^{2}}{2}\left[(1-2 \beta) \ddot{\mathbf{u}}^{t}+2 \beta \ddot{\mathbf{u}}^{t+1}\right] \\
& \dot{\mathbf{u}}^{t+1}=\dot{\mathbf{u}}^{t}+\Delta t\left[(1-\gamma) \ddot{\mathbf{u}}^{t}+\gamma \ddot{\mathbf{u}}^{t+1}\right]
\end{aligned}
$$

which depend on the coefficients $\beta$ and $\gamma$ and the time step $\Delta t$. The damping, elastic and external forces in Eq. (51) are weighted in two consecutive time steps by a coefficient $\alpha$ leading to:

$$
\mathbf{M} \ddot{\mathbf{u}}^{t+1}+(1+\alpha) \mathbf{C} \dot{\mathbf{u}}^{t+1}-\alpha \mathbf{C} \dot{\mathbf{u}}^{t}+(1+\alpha) \mathbf{K} \mathbf{u}^{t+1}-\alpha \mathbf{K} \mathbf{u}^{t}=(1+\alpha) \mathbf{F}^{t+1}-\alpha \mathbf{F}^{t}
$$

We can rearrange Eq. (52) in order to obtain $\ddot{\mathbf{u}}^{t+1}$ and $\dot{\mathbf{u}}^{t+1}$ as a function of the displacements $\mathbf{u}^{t+1}$ and all the variables evaluated in the previous time step as:

$$
\begin{aligned}
& \dot{\mathbf{u}}^{t+1}=b_{4}\left(\mathbf{u}^{t+1}-\mathbf{u}^{t}\right)-b_{5} \dot{\mathbf{u}}^{t}-b_{6} \ddot{\mathbf{u}}^{t} \\
& \ddot{\mathbf{u}}^{t+1}=b_{1}\left(\mathbf{u}^{t+1}-\mathbf{u}^{t}\right)-b_{2} \dot{\mathbf{u}}^{t}-b_{3} \ddot{\mathbf{u}}^{t}
\end{aligned}
$$

where the constants $b_{i}$ are:

$$
\begin{array}{ccc}
b_{1}=\frac{1}{\beta \Delta t^{2}} & b_{2}=\frac{1}{\beta \Delta} & b_{3}=1-\frac{1}{2 \beta} \\
b_{4}=\gamma \Delta t b_{1} & b_{5}=1+\gamma \Delta t b_{2} & b_{6}=\Delta t\left(1+\gamma b_{3}-\gamma\right)
\end{array}
$$

Then, substituting them in (53) results in:

$$
\mathbf{A} \mathbf{u}^{t+1}=\mathbf{b}
$$


where

$$
\begin{aligned}
\mathbf{A} & =(1+\alpha)\left[\mathbf{K}+b_{4} \mathbf{C}\right]+b_{1} \mathbf{M} \\
\mathbf{b} & =(1+\alpha) \mathbf{F}^{t+1}-\alpha \mathbf{F}^{t}+\alpha\left[\mathbf{K} \mathbf{u}^{t}+\mathbf{C} \dot{\mathbf{u}}^{t}\right]+\mathbf{M}\left[b_{1} \mathbf{u}^{t}-b_{2} \dot{\mathbf{u}}^{t}-b_{3} \ddot{\mathbf{u}}^{t}\right] \\
& +(1+\alpha) \mathbf{C}\left[b_{4} \mathbf{u}^{t}-b_{5} \dot{\mathbf{u}}^{t}-b_{6} \ddot{\mathbf{u}}^{t}\right]
\end{aligned}
$$

Once the displacements have been obtained by solving the system (56), it is easy to compute the velocities and accelerations from Eq. (54).

\section{Appendix B. List of symbols}

For the sake of clarity, in the table below we list all the symbols used throughout the paper in order of appearance:

$\begin{array}{ll}\mathbf{q}_{c}, \mathbf{q}_{b} & \text { Vector of degrees of freedom for cable and bar elements. } \\ \mathbf{r}(x, y, z) & \text { Absolute position vector and coordinates. } \\ \chi, s & \text { Local reference and deformed element coordinates. } \\ \xi & \text { Normalized local element coordinate. } \\ l_{r e f} & \text { Undeformed element length. } \\ \mathbf{N}_{c}, \mathbf{N}_{b} & \text { Shape functions matrix for cable and bar elements. } \\ m_{i}, c_{i}, k_{i}, i=1,2,3 & \text { Mass, damping and stiffness parameters of the pantograph model. } \\ k_{h} & \text { Penalty interaction stiffness. } \\ f_{i} & \text { Interaction force value. } \\ z_{1} & \text { Absolute vertical position of the degree of freedom 1 of the pantograph. } \\ z_{c w} & \text { Absolute vertical position of the contact wire at the contact point. } \\ \Omega, \Omega^{e} & \text { Catenary and element spatial domains. } \\ N_{e} & \text { Total number of finite elements. } \\ \delta W_{i n t}^{e}, \delta W_{e x t}^{e} & \text { Elemental virtual work produced by internal and external forces. } \\ E & \text { Young's modulus. } \\ A & \text { Cross-sectional area. } \\ I & \text { Second moment of area. } \\ \rho & \text { Density. } \\ \mathbf{g}, g & \text { Gravitational force vector and gravitational constant. } \\ \varepsilon_{a} & \text { Axial strain. }\end{array}$


$\kappa$

$\mathbf{f}_{i n t}, \mathbf{f}_{e x t}$

$\mathbf{r}_{0}$

u

$u, v, w$

$\mathbf{n}_{d}, \hat{\mathbf{n}}_{d}$

$\varepsilon_{a, 0}, T_{0}$

$\mathbf{k}_{d}, k_{d}$

$\mathbf{c}_{d}$

$\alpha_{r}, \beta_{r}$

$\mathbf{M}_{\text {cat }}, \mathbf{C}_{\text {cat }}, \mathbf{K}_{\text {cat }}$

$\mathbf{u}_{c a t}, \dot{\mathbf{u}}_{c a t}, \ddot{\mathbf{u}}_{c a t}$

$\mathbf{M}_{\text {pan }}, \mathbf{C}_{\text {pan }}, \mathbf{K}_{\text {pan }}$

$\mathbf{w}_{\text {pan }}, \dot{\mathbf{w}}_{\text {pan }}, \ddot{\mathbf{w}}_{\text {pan }}$

$F_{\text {pan }}$

$w_{c w}$

$z_{0, c w}$

$\mathbf{k}_{i}, \mathbf{K}_{i}$

$\mathbf{s}_{i}, \mathbf{S}_{i}$

$\mathbf{M}, \mathbf{C}, \mathbf{K}, \mathbf{F}$

$\mathbf{u}^{0}, \dot{\mathbf{u}}^{0}, \ddot{\mathbf{u}}^{0}$

$\Delta t$

$N_{\text {stp }}$

$\alpha, \beta, \gamma$

$b_{i}, i=1, \ldots, 6$

$\mathbf{f}_{d}, \mathbf{s}_{d}$

$\delta_{d}, \delta_{0, d}$

$\square^{t}, \square_{j}$
A, b
$\mathbf{F}_{I C}$

Curvature.

Internal and external equivalent nodal forces.

Static equilibrium position of the catenary.

Vector of displacements from the reference position.

Displacements on directions $x, y$ and $z$.

Director vector of dropper $d$ at the static equilibrium position and its unitary counterpart.

Axial strain and tension of an element in the static equilibrium configuration.

Stiffness matrix and stiffness constant for dropper $d$.

Damping matrix for dropper $d$.

Proportional Rayleigh damping parameters.

Mass, damping and stiffness matrices of the catenary.

Displacements, velocities and accelerations of the catenary respect to the static equilibrium configuration.

Mass, damping and stiffness matrices of the pantograph.

Vertical displacements, velocities and accelerations of the pantograph d.o.f.

External uplift force applied to the pantograph.

Vertical displacement, respect to the static equilibrium position, of the contact wire particularized at the contact point.

Static equilibrium vertical position of the contact wire at the contact point.

Local and global interaction stiffness matrix.

Local and global interaction static force vector.

Global assembled mass, damping, stiffness matrices and force vector.

Initial displacements, velocities and accelerations of the global system.

Time step.

Total number of time steps.

HHT parameters.

Constant values depending on $\Delta t$ and the HHT parameters.

Total internal force and internal force in the static equilibrium position for dropper $d$.

Total elongation and elongation in the static equilibrium position for dropper d.

Any variable particularized at time step $t$ and iteration $j$.

Global time integration matrix and force vector.

Global initial conditions force vector. 
$\mathscr{D}, \mathscr{C}$

$\mathbf{A}_{\text {cat }}, \mathbf{A}_{\text {pan }}$

$\mathbf{L}_{c a t}, \mathbf{U}_{c a t}$

$\square^{*}$

$N_{d}, N_{s d}$

$\mathbf{F}_{k n}, \mathbf{F}_{i}, \mathbf{F}_{d}$

$\mathbf{u}_{F_{k n}}, \mathbf{u}_{F_{i}}, \mathbf{u}_{F_{d}}$

$w_{d, c w}^{*}$
Dropper slackening and contact loss state variables.

Time integration matrix for the catenary and the pantograph systems.

LU factorisation of $\mathbf{A}_{\text {cat }}$.

Unitary force or displacement produced by a unitary force.

Number of total and slackened droppers.

Vectors of known, interaction and slackened dropper correction forces.

Displacements produced by the known, interaction and slackened dropper correction forces.

Vertical displacement of the contact wire at the interaction point, produced by a unitary force applied on dropper $d$.

\section{Acknowledgements}

The authors would like to acknowledge the financial support received from the FPU program offered by the Ministerio de Educación, Cultura y Deporte under grant number (FPU13/04191), and also funding from the Universitat Politècnica de València and the Generalitat Valenciana (PROMETEO/2016/007).

\section{References}

[1] A. Shing and P. Wong, "Wear of pantograph collector strips," Proceedings of the Institution of Mechanical Engineers, Part F: Journal of Rail and Rapid Transit, vol. 222, no. 2, pp. 169-176, 2008.

[2] G. Bucca, A. Collina, R. Manigrasso, F. Mapelli, and D. Tarsitano, "Analysis of electrical interferences related to the current collection quality in pantograph-catenary interaction," Proceedings of the Institution of Mechanical Engineers, Part F: Journal of Rail and Rapid Transit, vol. 225, no. 5, pp. 483-500, 2011. 
[3] S. Bruni, J. Ambrosio, A. Carnicero, Y. H. Cho, L. Finner, M. Ikeda, S. Y. Kwon, J.P. Massat, S. Stichel, and M. Tur, "The results of the pantograph-catenary interaction benchmark," Vehicle System Dynamics, vol. 53, no. 3, pp. 412-435, 2015.

[4] UNE 50318, "Railway applications. Current collection systems. Validation of simulation of the dynamic interaction between pantograph and overhead contact line," European Committee for Electrotechnical Standarization, 2002.

[5] K. Manabe and Y. Fujii, "Overhead system resonance with multi-pantographs and countermeasures," Railway Technical Research Institute, Quarterly Reports, vol. 30, no. 4, 1989.

[6] G. Gilbert and H. Davies, "Pantograph motion on a nearly uniform railway overhead line," Proceedings of the Institution of Electrical Engineers, vol. 113, no. 3, pp. 485492, 1966.

[7] S. H. Kia, F. Bartolini, A. Mpanda-Mabwe, and R. Ceschi, "Pantograph-catenary interaction model comparison," in IECON 2010-36th Annual Conference on IEEE Industrial Electronics Society, pp. 1584-1589, IEEE, 2010.

[8] J. Ambrosio, F. Rauter, J. Pombo, and M. Pereira, "A flexible multibody pantograph model for the analysis of the catenary-pantograph contact," vol. 23, pp. 1-27, 2011.

[9] O. Lopez-Garcia, A. Carnicero, and J. Maroño, "Influence of stiffness and contact modelling on catenary-pantograph system dynamics," Journal of Sound and Vibration, vol. 299, no. 4, pp. 806-821, 2007.

[10] A. Facchinetti, L. Gasparetto, and S. Bruni, "Real-time catenary models for the hardware-in-the-loop simulation of the pantograph-catenary interaction," Vehicle System Dynamics, vol. 51, no. 4, pp. 499-516, 2013.

[11] J. R. Jimenez-Octavio, A. Carnicero, C. Sanchez-Rebollo, and M. Such, "A moving mesh method to deal with cable structures subjected to moving loads and its application to the catenary-pantograph dynamic interaction," Journal of Sound and Vibration, vol. 349, pp. 216-229, 2015.

[12] S. Gregori, M. Tur, E. Nadal, F. Fuenmayor, and F. Chinesta, "Parametric model for the simulation of the railway catenary system static equilibrium problem," Finite Elements in Analysis and Design, vol. 115, pp. 21-32, 2016. 
[13] R. Zhang, P. V. Lauenstein, and B. M. Phillips, "Real-time hybrid simulation of a shear building with a uni-axial shake table," Engineering Structures, vol. 119, pp. 217-229, 2016.

[14] M. Matar, D. Paradis, and R. Iravani, "Real-time simulation of modular multilevel converters for controller hardware-in-the-loop testing," IET Power Electronics, vol. 9, no. 1, pp. 42-50, 2016.

[15] R. Conti, E. Meli, and A. Ridolfi, "A full-scale roller-rig for railway vehicles: multibody modelling and hardware in the loop architecture," Multibody System Dynamics, vol. 37, no. 1, pp. 69-93, 2016.

[16] W. Zhang, G. Mei, X. Wu, and Z. Shen, "Hybrid simulation of dynamics for the pantograph-catenary system," Vehicle System Dynamics, vol. 38, no. 6, pp. 393-414, 2002.

[17] A. Collina and S. Bruni, "Numerical simulation of pantograph-overhead equipment interaction," Vehicle System Dynamics, vol. 38, no. 4, pp. 261-291, 2002.

[18] A. Shabana, "Computer implementation of the absolute nodal coordinate formulation for flexible multibody dynamics," Nonlinear Dynamics, vol. 16, pp. 293-306, 1998.

[19] J. Gerstmayr and A. A. Shabana, "Analysis of thin beams and cables using the absolute nodal co-ordinate formulation," Nonlinear Dynamics, vol. 45, no. 1-2, pp. 109$130,2006$.

[20] J. Seo, A. S. Kim, I. Jung, T. Park, J. Mok, Y. Kim, and J. Chai, "Dynamic analysis of a pantograph-catenary system using absolute nodal coordinates," Vehicle System Dynamics, vol. 44, no. 8, pp. 615-630, 2006.

[21] J. H. Lee and T. W. Park, "Development of a three-dimensional catenary model using cable elements based on absolute nodal coordinate formulation," Journal of mechanical science and technology, vol. 26, no. 12, pp. 3933-3941, 2012.

[22] I. Romero, "A comparison of finite elements nonlinear beams: the absolute nodal coordinate and geometrically exact formulations," Multibody System Dynamics, vol. 20, pp. 51-68, 2008. 
[23] J. Gerstmayr and H. Irschik, "On the correct representation of bending and axial deformation in the absolute nodal coordinate formulation with an elastic line approach," Journal of Sound and Vibration, vol. 318, pp. 461-487, 2008.

[24] A. Collina and A. L. C. M. Carnevale, "Effect of collector deformable modes in pantograph-catenary dynamic interaction," Proceedings of the Institution of Mechanical Engineers, Part F: Journal of Rail and Rapid Transit, vol. 223, no. 1, pp. 1-14, 2009.

[25] Y. H. Cho, "Spops statement of methods," Vehicle System Dynamics, vol. 53, no. 3, pp. 329-340, 2015.

[26] H. Lankarani and P. E. Nikravesh, "A contact force model with hysteresis damping for impact analysis of multibody systems," Journal of Mechanical Design, vol. 112, no. 3, pp. 369-376, 1990.

[27] M. Tur, E. García, L. Baeza, and F. Fuenmayor, "A 3D absolute nodal coordinate finite element model to compute the initial configuration of a railway catenary," Engineering Structures, vol. 71, pp. 234-243, 2014.

[28] M. Berzeri and A. Shabana, "Development of simple models for the elastic forces in the absolute nodal coordinate formulation," Journal of Sound and Vibration, vol. 235, no. 4, pp. 539-565, 2000.

[29] H. M. Hilber, T. J. Hughes, and R. L. Taylor, "Improved numerical dissipation for time integration algorithms in structural dynamics," Earthquake Engineering \& Structural Dynamics, vol. 5, no. 3, pp. 283-292, 1977.

[30] J. Ambrósio, J. Pombo, P. Antunes, and M. Pereira, "Pantocat statement of method," Vehicle System Dynamics, vol. 53, no. 3, pp. 314-328, 2015.

[31] E. Cuthill and J. McKee, "Reducing the bandwidth of sparse symmetric matrices," in Proceedings of the 1969 24th national conference, pp. 157-172, ACM, 1969. 